\title{
Hamilton's Formalism for Systems with Constraints
}

\author{
Andreas W. Wipf \\ Institut für Theoretische Physik \\ Eidgenössische Technische Hochschule \\ Hönggerberg, CH-8093 Zürich, Switzerland \\ Lectures given at the Seminar "The Canonical Formalism in Classical \\ and Quantum General Relativity", Bad Honnef, September 1993 \\ ETH-TH/93-48
}




\begin{abstract}
The main goal of these lectures is to introduce and review the Hamiltonian formalism for classical constrained systems and in particular gauge theories. Emphasis is put on the relation between local symmetries and constraints and on the relation between Lagrangean and Hamiltonian symmetries.
\end{abstract}




\section{Contents}

\begin{tabular}{lll}
\hline 1 & Introduction & 2
\end{tabular}

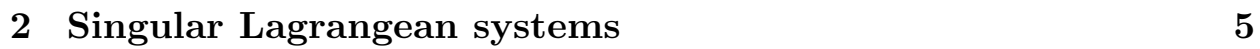

2.1 Singular Lagrangeans . . . . . . . . . . . . . . . . . 5

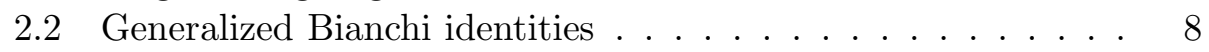

3 Hamilton's Formalism for Constraint Systems. 11

$3.1 \quad$ Primary constraints . . . . . . . . . . . . 11

3.2 Legendre transformation . . . . . . . . . . . . . . . . . . . . . 14

3.3 Dirac-Bergman algorithm . . . . . . . . . . . . . . . 16

3.4 $\quad$ First and second class constraints . . . . . . . . . . . . . . . 17

4 Abelian Chern-Simons Theory with Sources 20

\begin{tabular}{|lll}
5 & The reduced phase space. & 24
\end{tabular}

5.1 Second class constraints and Dirac bracket . . . . . . . . . . . 24

5.2 First class constraints and gauge transformations . . . . . . . 27

5.3 Mixed second and first class constraints . . . . . . . . . . 31

5.4 Gauge fixing of Chern-Simons theory . . . . . . . . . . . . . . 31

5.5 First order action principle and symmetries . . . . . . . . . 34

\begin{tabular}{lll}
\hline 6 & Yang-Mills Theories & 36
\end{tabular}

7 Lagrangean Symmetries of First-Class Hamiltonian Systems 40

7.1 The relativistic particle $\ldots \ldots \ldots \ldots$. . . . . . . . 40

7.2 Hamiltonian vs. Lagrangean symmetries . . . . . . . . . . . 41 


\section{Chapter 1}

\section{Introduction}

All fundamental field theories in physics are invariant with respect to some group of local symmetry transformations. For Yang-Mills theories these are the gauge transformations, for string theory and gravity space-time diffeomorphisms and for supersymmetric theories local supersymmetry transformations. In such theories which are called gauge theories or more generally singular systems, the local symmetry relates different solutions stemming from the same initial conditions and the general solution of the equations of motion contains arbitrary time-dependent functions. Hence there is a continuous set of accelerations which belong to the same initial position and velocity and we expect that all accelerations correspond only to a subset of initial conditions. This subset is defined by the Lagrangean constraints?. Hence all gauge theories are systems with constraints. In the Hamiltonian formalism this means that there are conditions on the allowed initial momenta and positions. These conditions must then be conserved by the time evolution, and this requirement may lead to further constraints.

One should distinguish between gauge theories with internal symmetries and those which are generally covariant.

For the former all constraints are linear in the momenta and the Hamiltonian does not vanish. The local symmetry transformations are generated by the first class constraints.

For generally covariant theories at least one constraint is quadratic in the momentaf and there are canonical variables for which the Hamiltonian $H$ itself is a constraint, usually called superhamiltonian. This leads to the

\footnotetext{
${ }^{1}$ this statement is made more precise by the off-shell Bianchi identities

${ }^{2}$ topological field theories are exceptional in this respect
} 
question whether $H$ generates the dynamical time-evolution or kinematical local symmetries as the other first class constraints. We will hear more about this problem of interpreting time in the lectures of R. Baig and P. Hajicek.

Attempts to handle constrained systems date back more than forty years. In his classical works Dirac set up a formalism to treat such systems selfconsistently 17]. Later Bergman et.al. in a series of papers investigated the connection between constraints and invariances [3, 10, 12]. After the introduction of Grassmann variables to describe fermions [8], the formalism has been extended to include fields with half-integer spins [23, 13, 9]. The development culminated with the advent of the elegant and powerful BRST formalism [6]. These and other classical results have been a prerequisite for the quantization of gauge theories both in the path integral formalism [19, 5] and in the functional Schrödinger picture [43, 31, 32].

Besides the classical lectures of Dirac 18 there are several excellent reviews on the treatment of constrained systems. Some focus more on systems with a finite number of degrees of freedom [41], others on field theories [28] and some on both 42, 25, 29. For generally covariant theories you may consult 24].

In these hopefully selfcontained lectures I applied the developed formalism at various stages to the abelian Chern-Simons model with sources [27, 36, 47]. In this way the reader may become acquainted with the constrained dynamics by way of example. There are several aspects I could not cover in these lectures, especially the inclusion of fermions which leads to graded Poisson-structures [23, 13] and the popular BRST formalism [6]. Generally covariant theories and in particular gravity are covered by the lectures of R. Baig and N. Giulini on classical gravity and the other contributions to the proceedings.

In the first part of these lectures I followed the conventional discussion of constrained systems. The second chapter is devoted to singular Lagrangean systems. I discuss the off-shell Bianchi identities and show that all gauge theories are constrained systems. In chapter three some important facts about constrained Hamiltonian systems are reviewed and discussed. In particular primary/secondary and first/second class constraints, the generalized Legendre transformation and the Dirac-Bergman algorithm are introduced. The general formalism is applied to the abelian Chern-Simons theory in chapter four. Then we introduce the reduced phase space for first and second class systems. Here the important Dirac brackets for second class (SC) systems, the concept of observables and gauge transformations for first class (FC) 
systems and the first order formalism for mixed SC and FC systems are discussed. Again I apply the general results to the abelian Chern-Simons theory and show that the only observables are the Wilson-loops. In the last chapter I investigate the relation between Lagrangean symmetries and the Hamiltonian gauge transformations generated by the first class constraint [35. We shall see that the latter must be supplemented by transformations which vanish on-shell in order to recover the Lagrangean symmetries. Also, I will discuss for which theories the equations of motion follow from the local symmetries. Some of the results in this last chapter are new and have not previously been published. We feel that the results offered are somewhat novel.

I am indepted to V. Mukhanov for sharing with me many insights and constributing to these notes. 


\section{Chapter 2}

\section{Singular Lagrangean systems}

\subsection{Singular Lagrangeans}

In these lectures I consider systems whose dynamics can be derived from Hamilton's variational principle. I assume that all Lagrangeans depend at most on first derivatives, up to divergence terms. For higher derivative theories, and in particular for higher derivative gravity, see [24]. I use local coordinates, unless I am forced to address global questions, e.g. the Gribov problem or the role of topologically nontrivial field configurations.

With these assumptions the classical trajectories of a system with $N$ degrees of freedom make the action

$$
S=\int_{t_{1}}^{t_{2}} L\left(q^{i}, \dot{q}^{i}\right) d t \quad, \quad i=1, \ldots, N
$$

stationary under variations $\delta q(t)$ which vanish at the endpoints. The $q$ and $\dot{q}$ are local coordinates on the velocity phase space $T Q$. The necessary conditions for $S$ to be stationary are the Euler-Lagrange equations

$$
L_{i} \equiv-\frac{d}{d t}\left(\frac{\partial L}{\partial \dot{q}^{i}}\right)+\frac{\partial L}{\partial q^{i}}=0
$$

which can be rewritten as

$$
L_{i}=-\frac{\partial^{2} L}{\partial \dot{q}^{i} \partial \dot{q}^{j}} \ddot{q}^{j}-\frac{\partial^{2} L}{\partial \dot{q}^{i} \partial q^{j}} \dot{q}^{j}+\frac{\partial L}{\partial q^{i}} \equiv-W_{i j}(q, \dot{q}) \ddot{q}^{j}+V_{i}=0 .
$$

We see that the accelerations at a given time are uniquely determined by $(q, \dot{q})$ at that time only if the Hessian $\left(W_{i j}\right)$ can be inverted. Such systems are called regular. 
If, on the other hand, $\operatorname{det} W=0$, the accelerations and thus the time evolution will not be uniquely fixed by the $(q, \dot{q})$. Such systems are called singular [3]. For singular systems different time evolutions will stem from the same initial conditions.

The rank $R$ of $W$, which we assume for simplicity to be constant on $T Q$, being $R<N$ implies the existence of $M=N-R$ null-eigenvectors

$$
Y_{m}^{i}(q, \dot{q}) W_{i j}(q, \dot{q})=0 \quad, \quad m=1, \ldots, M .
$$

Contracting the E-L equations 2.3 with the $Y_{m}$ we get

$$
\phi_{m}(q, \dot{q}) \equiv Y_{m}^{i} V_{i}=0 \quad, \quad m=1, \ldots, M .
$$

These equations do not contain accelerations. Assume that $M^{\prime} \leq M$ relations

$$
\phi_{m^{\prime}}=0 \quad, \quad m^{\prime}=1, \ldots, M^{\prime},
$$

are functionally independent on the others, and the remaining ones are either dependent or identically fulfilled. The independent $\phi_{m^{\prime}}$ are the socalled Lagrange constraints.

For field theories the dynamics is described by functions $\varphi^{a}(x)$ of spacetime with values in a certain target space. The index $a$ may belong to an internal symmetry, it may be a spacetime index or both internal and spacetime index as in nonabelian gauge theories. When going from point mechanics to field theory one may think of replacing the disrete label $i$ by a continuous one $(a, \vec{x})$ :

$$
q^{i}(t)=q(t, i) \longrightarrow q(t, a, \vec{x})=\varphi^{a}(t, \vec{x})=\varphi^{a}(x) .
$$

Summations become integrals, e.g.

$$
\sum_{i} \dot{q}^{i} \dot{q}^{i} \longrightarrow \sum \int d \vec{x} \dot{\varphi}^{a}(\vec{x}) \dot{\varphi}^{a}(\vec{x})
$$

and functions of $(q, \dot{q})$ become functionals of $\varphi$ and $\dot{\varphi}$. Also, derivatives with respect to $q^{i}$ or $\dot{q}^{i}$ become functional derivatives, e.g.

$$
\frac{\partial L}{\partial \dot{q}^{i}} \longrightarrow \frac{\delta L}{\delta \dot{\varphi}^{a}(\vec{x})}
$$

The suitable velocity phase space $T Q$ is chosen so that the Lagrange-functional $L$ is continuous and sufficiently often differentiable. If the target space is 
linear one may choose a Banach space (typically a Sobolov space), otherwise one tries to model the theory on a $C^{k}$-Banach manifold [15, 34] since the implicit function theorem still applies then. Banach manifolds are modelled over Banach spaces and are straightforward generalizations of finitedimensional manifolds.

A functional on a Banach space $X$ is called continuous if

$$
\lim _{n \rightarrow \infty} F\left[\varphi_{n}\right]=F[\varphi] \quad \text { for } \quad X \ni \varphi_{n} \rightarrow \varphi .
$$

$F$ is called Frechet-differentiable at $\varphi$ if there exists a linear functional $F_{\varphi}^{\prime}$ such that

$$
\left|F[\varphi+\delta \varphi]-F[\varphi]-F_{\varphi}^{\prime}[\delta \varphi]\right|=o(\|\delta \varphi\|) \quad \text { for all } \quad\|\delta \varphi\| \rightarrow 0 .
$$

For local theories the Lagrangean has the form

$$
L[\varphi, \dot{\varphi}]=\int d \vec{x} \mathcal{L}\left(\varphi, \partial_{i} \varphi, \dot{\varphi}\right)
$$

with a Lagrangean density $\mathcal{L}$ depending only on the field and its derivatives at the same point. For such theories the Euler-Lagrange equations are

$$
L_{a} \equiv-\frac{\partial}{\partial t} \frac{\delta L}{\delta \dot{\varphi}^{a}}+\frac{\delta L}{\delta \varphi^{a}}=-\partial_{\mu} \frac{\partial \mathcal{L}}{\partial\left(\partial_{\mu} \varphi^{a}\right)}+\frac{\partial \mathcal{L}}{\partial \varphi^{a}}=0,
$$

where I adopted the common notation

$$
F_{\varphi}^{\prime}[\delta \varphi] \equiv \int \frac{\delta F}{\delta \varphi(x)} \delta \varphi(x)
$$

Rewriting the field equations as

$$
\begin{gathered}
L_{a}=-\frac{\partial^{2} \mathcal{L}}{\partial\left(\partial_{\mu} \varphi^{a}\right) \partial\left(\partial_{\nu} \varphi^{b}\right)} \partial_{\mu} \partial_{\nu} \varphi^{b}-\frac{\partial^{2} \mathcal{L}}{\partial\left(\partial_{\mu} \varphi^{a}\right) \partial \varphi^{b}} \partial_{\mu} \varphi^{b}+\frac{\partial \mathcal{L}}{\partial \varphi^{a}} \\
\equiv-W_{a b}^{\mu \nu} \partial_{\mu} \partial_{\nu} \varphi^{b}+V_{a}=0
\end{gathered}
$$

we can see that theories with $x^{0}$ taken as evolution parameter are regular if $W_{a b}^{00}$ is invertible and singular if it is not. For singular systems there exist (for each $\vec{x}$ ) $M=N-R$ null-vectors

$$
Y_{m}^{a}(\varphi, \partial \varphi) W_{a b}^{00}(\varphi, \partial \varphi)=0 \quad, \quad m=1, \ldots, M
$$


which lead to nontrivial and independent relations

$$
\phi_{m^{\prime}}(\varphi, \partial \varphi) \equiv Y_{m^{\prime}}^{a} V_{a}=0 \quad, \quad m^{\prime}=1, \ldots, M^{\prime} \leq M,
$$

involving only the fields and their first derivatives. These are the Lagrangean constraints.

How one proceeds for singular systems is neatly explained in [39, 42]. There are two points which have to be considered. Firstly the rank of the Hessian may decrease if one takes the independent constraints $(2.6,12)$ into account. This may lead to new independent constraints. Again the rank may decrease leading to further constraints, etc. This process terminates as soon as the rank does not change anymore.

Secondly one needs to check whether the constraints one has found after the above algebraic process has terminated are respected by the time evolution. These may lead to new constraints. Again and again differentiate newly emerging constraints until no new ones arise. Add those relations involving accelerations to those already present. Consistency of the old relations with the new ones may lead to further constraints. After all that one needs again to check whether the rank of the Hessian has changed. If this is the case one needs to start from the beginning etc.

\subsection{Generalized Bianchi identities}

If a theory possesses a local gauge invariance we may map solutions into solutions without affecting the initial conditions. Thus we expect that gauge theories are singular systems. Actually this follows from the generalized Bianchi identity [46, 44] which we derive next.

The point transformations

$$
\begin{aligned}
x^{\prime} & =x^{\prime}(x) \sim x+\delta x \\
\varphi^{\prime}\left(x^{\prime}\right) & =\varphi^{\prime}(\varphi(x), x) \sim \varphi(x)+\delta \varphi
\end{aligned}
$$

which leave the action invariant

$$
\int d^{d} x^{\prime} \mathcal{L}\left(\varphi^{\prime}, \partial^{\prime} \varphi^{\prime}, x^{\prime}\right)=\int d^{d} x \mathcal{L}(\varphi, \partial \varphi, x)
$$

form a group which we assume to be continuous. For transformations close to the identity $d^{d} x^{\prime}=d^{d} x\left(1+\partial_{\mu} \delta x^{\mu}\right)$, and the invariance 2.19 implies

$$
\delta \mathcal{L}+\mathcal{L} \partial_{\mu} \delta x^{\mu}=\partial_{\mu} \lambda^{\mu}
$$


with some $\lambda$. Using

$$
\delta \mathcal{L}=\frac{\partial \mathcal{L}}{\partial \varphi^{a}} \delta \varphi^{a}+\frac{\partial \mathcal{L}}{\partial\left(\partial_{\nu} \varphi^{a}\right)} \delta\left(\partial_{\nu} \varphi^{a}\right)+\partial_{\mu} \mathcal{L} \delta x^{\mu}
$$

it follows at once that

$$
\delta \mathcal{L}+\mathcal{L} \partial_{\mu} \delta x^{\mu}=\partial_{\mu}\left(\mathcal{L} \delta x^{\mu}\right)+\partial_{\mu}\left(\frac{\partial \mathcal{L}}{\partial\left(\partial_{\mu} \varphi^{a}\right)} \bar{\delta} \varphi^{a}\right)+L_{a} \bar{\delta} \varphi^{a},
$$

where the Euler derivatives $L_{a}$ have been defined in 2.13 and

$$
\bar{\delta} \varphi^{a}=\delta \varphi^{a}-\partial_{\mu} \varphi^{a} \delta x^{\mu} \sim \varphi^{a \prime}(x)-\varphi^{a}(x)
$$

is the infinitesimal difference of the old and the transformed files at the same point. We used that $\left[\bar{\delta}, \partial_{\mu}\right]=0$. Thus the gauge invariance implies

$$
\partial_{\mu}\left(\mathcal{L} \delta x^{\mu}+\frac{\partial \mathcal{L}}{\partial\left(\partial_{\mu} \varphi^{a}\right)} \bar{\delta} \varphi^{a}-\lambda^{\mu}\right)+L_{a} \bar{\delta} \varphi^{a}=0
$$

and these are the generalized Bianchi identities. Nowhere did we use the equation of motion and thus 2.24 are off-shell identities.

First assume that $S$ is invariant under global transformations forming a $n$-dimensional Lie-group. Then

$$
\lambda^{\mu}=\epsilon_{\alpha} \lambda^{\alpha \mu}, \quad \delta_{\epsilon} x^{\mu}=\epsilon_{\alpha} A^{\alpha \mu}, \quad \delta_{\epsilon} \varphi^{a}=\epsilon_{\alpha} B^{\alpha a},
$$

where the $\epsilon_{\alpha}, \alpha=1, \ldots, n$ are the constant parameters of the infinitesimal transformations. Inserting this into 2.24 and going on shell, $L_{a}=0$, we conclude

$$
\partial j^{\alpha \mu}=0, \quad \text { where } \quad j^{\alpha \mu}=\frac{\partial \mathcal{L}}{\partial\left(\partial_{\mu} \varphi^{a}\right)}\left(B^{\alpha a}-A^{\alpha \nu} \partial_{\nu} \varphi^{a}\right)+\mathcal{L} A^{\alpha \mu}-\lambda^{\alpha \mu},
$$

which is Noether's first theorem. When deriving 2.26 we imposed the equations of motion so that the currents are conserved only on-shell.

Let us now assume that the symmetry transformations are local. In that case the parameters become space-time dependent. Then 2.25 generalizes to

$$
\delta_{\epsilon} x^{\mu}=\epsilon_{\alpha} A^{\alpha \mu} \quad, \quad \delta_{\epsilon} \varphi^{a}=\epsilon_{\alpha} B^{\alpha a}+\partial_{\mu} \epsilon_{\alpha} C^{\alpha a \mu},
$$

where the $\epsilon_{\alpha}(x)$ parametrize the infinitesimal local gauge transformations and $B$ and $C$ are the socalled descriptors [3], which in general depend on the 
fields and their derivatives. I assumed that no second or higher derivatives of $\epsilon$ enter because this covers most interesting examples. With

$$
\bar{\delta}_{\epsilon} \varphi^{a}=\epsilon_{\alpha}\left(B^{\alpha a}-\partial_{\mu} \varphi^{a} A^{\alpha \mu}\right)+\partial_{\mu} \epsilon_{\alpha} C^{\alpha a \mu}
$$

the integrated form of 2.24, after a partial integration, reads

$$
0=\int \epsilon_{\alpha}\left[L_{a}\left(B^{\alpha a}-\partial_{\mu} \varphi^{a} A^{\alpha \mu}\right)-\partial_{\mu}\left(L_{a} C^{\alpha a \mu}\right)\right] .
$$

Since it must hold for arbitrary functions $\epsilon_{\alpha}$ this implies that the expression between the square brackets must vanish. Inserting $L_{a}$ from 2.1 we end up with

$$
\begin{aligned}
0= & L_{a}\left(B^{\alpha a}-\partial_{\mu} \varphi^{a} A^{\alpha \mu}-\partial_{\mu} C^{\alpha a \mu}\right)-C^{\alpha a \mu} \partial_{\mu} V_{a} \\
& +C^{\alpha a \mu}\left(\partial_{\mu} W_{a b}^{\rho \sigma \rho} \partial_{\rho} \partial_{\sigma} \varphi^{b}+W_{a b}^{\rho \sigma} \partial_{\mu} \partial_{\rho} \partial_{\sigma} \varphi^{b}\right) .
\end{aligned}
$$

Since these are off-shell identities we conclude

$$
C^{\alpha a(\mu} W_{a b}^{\rho \sigma)}=0
$$

where the brackets around the indices mean symmetrization. In particular, descriptors $C^{\alpha a 0}$ which are not identically zero are null-eigenvectors of the Hessian,

$$
C^{\alpha a 0} W_{a b}^{00}=0
$$

and render the system singular. If all $C^{\alpha a \mu}$ vanish, then 2.2 reduces to

$$
0=\left(B^{\alpha a}-\partial_{\rho} \varphi^{a} A^{\alpha \rho}\right) L_{a} \Longrightarrow\left(B^{\alpha a}-A^{\alpha \rho} \partial_{\rho} \varphi^{a}\right) W_{a b}^{(\mu \nu)}=0 .
$$

Thus, if $C \equiv 0$ but the $B^{\alpha a}-A^{\alpha \rho} \partial_{\rho} \varphi^{a}$ are not identically zero, we conclude again that the system is singular. So we have the important result that gauge theories are necessarily singular. However, the converse is not true. Not all conceivable singular systems are gauge theories. 


\section{Chapter 3}

\section{Hamilton's Formalism for Constraint Systems.}

\subsection{Primary constraints}

The departing point for the Hamiltonian formalism is to define the canonical momenta (densities) by

$$
p_{i}=\frac{\partial L}{\partial \dot{q}^{i}}(q, \dot{q}) \quad \text { resp. } \quad \pi_{a}(\vec{x})=\frac{\delta L}{\delta \dot{\varphi}^{a}(\vec{x})},
$$

where we assume that $L \in C^{2}(T Q)$. Only if

$$
W_{i j}=\frac{\partial p_{i}}{\partial \dot{q}^{j}} \quad \text { resp. } \quad W_{a b}^{00}(\vec{x}, \vec{y})=\frac{\delta \pi_{a}(\vec{x})}{\delta \dot{\varphi}^{b}(\vec{y})}
$$

is invertible can this relation be solved for all velocities in terms of the phase space variables, $\dot{q}=\dot{q}(q, p)$ resp. $\dot{\varphi}=\dot{\varphi}(\varphi, \pi)$ 円. In the other case not all momenta 3.1 are independent, but there are some relations

$$
\phi_{m}(q, p)=0 \quad \text { resp. } \quad \phi_{m}(\varphi, \pi)=0 \quad, \quad m=1, \ldots, M
$$

that follow from the definition 3.1 of the momenta. In these lectures I assume that the constraints 3.3 are independent.

In the following we restrict ourselves to finite dimensional systems and only comment on the related results for field theories. The corresponding

\footnotetext{
${ }^{1}$ for field theories we assume $T Q$ to be a Banach manifold so that the inverse function theorem applies
} 
field theoretical formalae, if they apply, are obtained if one uses deWitt's condensed notation [16] in which $i$ becomes a composite index as explained above and in chapter 7 .

The conditions 3.3 are the $M=N-R$ primary constraints. They define the $2 N-M$-dimensional primary constraint surface, denoted by $\Gamma_{p}$. The equations of motions have not been used to derive them and they imply no restriction on the $(q, \dot{q})$. 3.1 maps the $2 N$-dimensional velocity phase space $T Q$ to the lower-dimensional submanifold $\Gamma_{p}$ in the momentum phase space $\Gamma$. Hence the inverse images of a given point in $\Gamma_{p}$ form a manifold of dimension $M$.

To pass to the Hamiltonian formalism we impose some regularity conditions on the primary constraints. They can be alternatively formulated as:

1. the independent functions $\phi_{m}, m=1, \ldots, M$ can be locally taken as the first $M$ coordinates of a new, regular, coordinate system in the vicinity of $\Gamma_{p}$.

2. The gradients $d \phi_{1}, \ldots, d \phi_{M}$ are locally linearly independent on $\Gamma_{p}$; i.e., $d \phi_{1} \wedge \ldots \wedge d \phi_{M} \neq 0$ on $\Gamma_{p}$.

For example, if $\phi$ is an admissible constraint, $\phi^{2}$ is not, since $d\left(\phi^{2}\right)=2 \phi d \phi=0$ on $\Gamma_{p}$. If the constraints are regular the following properties hold.

Theorem 1 If a smooth function $F(q, p)$ vanishes on $\Gamma_{p}$, then $F=f^{m} \phi_{m}$ for some functions $f^{m}$.

Theorem 2 If $\lambda_{i} \delta q^{i}+\mu^{i} \delta p_{i}=0$ for arbitrary variations $\delta q^{i}, \delta p_{i}$ tangent to the constraint surface, then

$$
\lambda_{i}=u^{m} \frac{\partial \phi_{m}}{\partial q^{i}} \quad \text { and } \quad \mu^{i}=u^{m} \frac{\partial \phi_{m}}{\partial p_{i}} \quad \text { on } \quad \Gamma_{p}
$$

for some $u^{m}$.

Before proving these two important theorems it is useful to distinguish between weak and strong equations. A function $F(q, p)$ defined in the neighbourhood of $\Gamma_{p}$ is called weakly zero if

$$
\left.F\right|_{\Gamma_{p}}=0 \Longleftrightarrow F \approx 0
$$


and strongly zero if

$$
\left.F\right|_{\Gamma_{p}}=0 \quad \text { and }\left.\quad\left(\frac{\partial F}{\partial q^{i}}, \frac{\partial F}{\partial p_{i}}\right)\right|_{\Gamma_{p}=0} \Longleftrightarrow F \simeq 0 .
$$

These definitions are useful since the equations of motion contain gradients of functions on $\Gamma_{p}$. The primary constraint surface can itself be defined by weak equations. We have

$$
\phi_{m} \approx 0 \quad \text { but } \quad \phi_{m} \neq 0
$$

because of our regularity conditions on the constraints.

Since $\nabla_{x}\left(f^{m} \phi_{m}\right) \approx f^{m} \nabla_{x} \phi_{m}$, where $x=(q, p)$ denotes the phase space coordinates, the first theorem implies

Lemma $1 F \approx 0 \Longrightarrow F-f^{m} \phi_{m} \simeq 0$ for some functions $f^{m}$.

To prove the first theorem we choose the independent constraints $\phi_{m}$ as first coordinates of a regular coordinate system $x=(\phi, \tilde{x})$ in the neighbourhood of $\Gamma_{p}$. Since $F(0, \tilde{x})=0$ we have

$$
F(\phi, \tilde{x})=\int_{0}^{1} \frac{d}{d \tau} F(\tau \phi, \tilde{x}) d \tau=\phi_{m} \int_{0}^{1} F,_{m}(\tau \phi, \tilde{x}) d \tau
$$

and thus

$$
F=f^{m} \phi_{m} \quad \text { with } \quad f^{m}=\int_{0}^{1} F_{, m}(\tau \phi, \tilde{x}) d \tau .
$$

This proves theorem 1 in the neighbourhood $U$ of any point on $\Gamma_{p}$. We cover the neighbourhood of $\Gamma_{p}$ by open sets $U_{i}$, on each of which theorem 1 applies. Together with the open sets $V_{k}$ on which $\phi_{k} \neq 0$ the $U_{i}$ cover the whole phase space. On $V_{k}$ we can set $F=\left(F / \phi_{k}\right) \phi_{k}$ and theorem 1 holds there. Finally, to guarantee that the $f^{m}$ are the same on the overlap of $U_{i}$ and $U_{i^{\prime}}$ one uses a finite partition of unity.

Theorem 2 follows immediately from the regularity condition which implies that at a given point $x$ on $\Gamma_{p}$ a basis of $T_{x} \Gamma_{p}$ (the vectors tangent to $\Gamma_{p}$ at $x$ ), together with the gradients $\nabla_{x} \phi_{m}$ form a basis of $T_{x} \Gamma$. The assumption in theorem 2 means that $(\lambda, \mu)$ are orthogonal to $T_{x} \Gamma_{p}$. Thus it must be a linear combination of the gradients $\nabla_{x} \phi_{m}$. 
For field theories one finds

$$
F[\phi, \tilde{x}] \approx 0 \Rightarrow F=\int f^{m} \phi_{m} \quad, \quad f^{m}(\vec{x})=\int d \tau \frac{\delta F}{\delta \phi_{m}(\vec{x})}[\tau \phi, \tilde{x}]
$$

and a weakly vanishing functional is a linear combination of smeared constraints. The testfunctions should lie in the space dual to the space of the constraints [7].

\subsection{Legendre transformation}

The canonical Hamiltonian

$$
H=\dot{q}^{i} p_{i}-L \quad \text { resp. } \quad H=\int d \vec{x} \pi_{a}(\vec{x}) \dot{\varphi}^{a}(\vec{x})-L=\int d \vec{x} \mathcal{H}
$$

has the remarkable property that $\dot{q}$ enters $H$ only through the combination $p(q, \dot{q})$. This follows from

$$
\begin{aligned}
\delta H & =\dot{q}^{i} \delta p_{i}+\delta \dot{q}^{i} p_{i}-\delta \dot{q}^{i} \frac{\partial L}{\partial \dot{q}^{i}}-\delta q^{i} \frac{\partial L}{\partial q^{i}} \\
& =\dot{q}^{i} \delta p_{i}-\delta q^{i} \frac{\partial L}{\partial q^{i}}
\end{aligned}
$$

which shows that $H$ is a function of $p$ and $q$ only. Here $\delta p$ is to be regarded as linear combination of $\delta q$ and $\delta \dot{q}$ so that $\delta q, \delta p$ are tangent to $\Gamma_{p} . H$ is only defined on $\Gamma_{p}$ since we used the constraints. We would like to extend the formalism to the whole phase space $\Gamma$.

The equation 3.2 can be rewritten as

$$
\left(\frac{\partial H}{\partial q^{i}}+\frac{\partial L}{\partial q^{i}}\right) \delta q^{i}+\left(\frac{\partial H}{\partial p_{i}}-\dot{q}^{i}\right) \delta p_{i}=0
$$

with variations tangent to $\Gamma_{p}$. $H$ may be the restriction to the hypersurface $\Gamma_{p}$ of a function $\tilde{H}$ defined all over phase space. Then 3.13 holds with $H$ replaced by $\tilde{H}$. Applying theorem 2 we conclude that

$$
\begin{aligned}
\dot{q}^{i} & \approx \frac{\partial \tilde{H}}{\partial p_{i}}+u^{m} \frac{\partial \phi_{m}}{\partial p_{i}} \\
-\frac{\partial L}{\partial q^{i}} & \approx \frac{\partial \tilde{H}}{\partial q^{i}}+u^{m} \frac{\partial \phi_{m}}{\partial q^{i}} .
\end{aligned}
$$

The first set of relations enables us to recover the velocities from the $(q, p) \in$ 
$\Gamma_{p}$ and the parameters $u^{m}$. Because of the regularity conditions on the constraints two different $u$ yield different $\dot{q}$ and the first relation permits us to express $u$ as function of $q$ and $\dot{q}$. This way one obtains an invertible Legendre transformation from the $2 N$-dimensional velocity phase space to the $2 N$ dimensional space $\Gamma_{p} \times\left\{u^{m}\right\}$ :

$$
p_{i}=\frac{\partial L}{\partial \dot{q}^{i}}(q, \dot{q}) \quad \text { and } \quad u^{m}=u^{m}(q, \dot{q})
$$

with inverse transformation

$$
\dot{q}^{i}=\frac{\partial \tilde{H}}{\partial p_{i}}+u^{m} \frac{\partial \phi_{m}}{\partial p_{i}} \quad \text { and } \quad \phi_{m}(q, p)=0 .
$$

We had to extend the Hamiltonian, which was originally defined only on $\Gamma_{p}$, to a neighbourhood of $\Gamma_{p}$. According to theorem 1 two possible extensions differ by a term $c^{m} \phi_{m}$. Thus the formalism should be unchanged by the replacement

$$
\tilde{H} \longrightarrow \tilde{H}+c^{m}(q, p) \phi_{m}
$$

Indeed, making this transformation in 3.2 just shifts the $u$ to $u+c$.

Finally, the relations 3.2 allow us to rewrite the equation of motion 2.2 in the equivalent Hamiltonian form

$$
\dot{q}^{i} \approx \frac{\partial H}{\partial p_{i}}+u^{m} \frac{\partial \phi_{m}}{\partial p_{i}} \quad \text { and } \quad \dot{p}_{i} \approx-\frac{\partial H}{\partial q^{i}}-u^{m} \frac{\partial \phi_{m}}{\partial q^{i}}
$$

where we dropped the tilde atop $H$. The Lagrangean equations of motion 2.2 are equivalent to 3.18. The phase space function

$$
H_{p} \equiv H+u^{m} \phi_{m}
$$

os the primary Hamiltonian.

Introducing the Poisson bracket of two phase space functions

$$
\begin{aligned}
\{F, G\} & \equiv \frac{\partial F}{\partial q^{i}} \frac{\partial G}{\partial p_{i}}-\frac{\partial F}{\partial p_{i}} \frac{\partial G}{\partial q^{i}} \quad \text { resp. } \\
\{F, G\} & \equiv \int d \vec{x}\left(\frac{\delta F}{\delta \varphi^{a}(\vec{x})} \frac{\delta G}{\delta \pi_{a}(\vec{x})}-\frac{\delta F}{\delta \pi_{a}(\vec{x})} \frac{\delta G}{\delta \varphi^{a}(\vec{x})}\right)
\end{aligned}
$$

and using $u^{m} \nabla_{x} \phi_{m} \approx \nabla_{x}\left(u^{m} \phi_{m}\right)$, the Hamiltonian equations of motion can be rewritten as 


$$
\begin{aligned}
& \dot{q}^{i} \approx\left\{q^{i}, H_{p}\right\} \approx\left\{q^{i}, H\right\}+\left\{q^{i}, \phi_{m}\right\} u^{m} \\
& \dot{p}_{i} \approx\left\{p_{i}, H_{p}\right\} \approx\left\{p_{i}, H\right\}+\left\{p_{i}, \phi_{m}\right\} u^{m} .
\end{aligned}
$$

Besides there are still the equations defining $\Gamma_{p}$ :

$$
\phi_{m}(q, p)=0 .
$$

For an any phase-space function $F(q, p)$ the time evolution follows then from

$$
\dot{F} \approx\left\{F, H_{p}\right\} \approx\{F, H\}+u^{m}\left\{F, \phi_{m}\right\} .
$$

\subsection{Dirac-Bergman algorithm}

As in the Lagrangean formalism the constraints must be consistent with the time evolution. If initially $(q, p)$ is on $\Gamma_{p}$ it should remain there at later times. This means that the equations of motion should preserve the constraints and this gives rise to the consistency conditions [17, 3]

$$
\dot{\phi}_{m} \approx\left\{\phi_{m}, H\right\}+\left\{\phi_{m}, \phi_{n}\right\} u^{n} \equiv h_{m}+C_{m n} u^{n} \approx 0 .
$$

For non-admissable Lagrangeans these relations will be inconsistent. As an example take $L=\dot{q}-q$ which leads to $H=q$ and $\phi=p-1$ so that 3.24 reads $1 \approx 0$. For such inconsistent models the action has no stationary points and we shall exclude them.

To discuss the consistency relations 3.24 we distinguish the two following cases:

i) $\operatorname{det} C \not 0$ :

In this case $u$ is uniquely fixed by 3.24 to be $u^{n} \approx C^{n m} h_{m}$, where $C^{n m}$ is the inverse of $C_{n m}$. The time evolution 3.23 of a phase space function becomes

$$
\dot{F} \approx\{F, H\}-\left\{F, \phi_{m}\right\} C^{m n}\left\{\phi_{n}, H\right\} .
$$

No additional conditions appear. For any initial data $(q, p)$ on $\Gamma_{p}$ the time evolution stemming from 3.25 is unambiguous and stays on $\Gamma_{p}$.

ii) $\operatorname{det} C \approx 0$ :

In this case $u$ is not fixed and 3.24 is only solvable if $h_{m} w_{a}^{m} \approx 0$ for all left null-eigenvectors $w_{a}$ of $C$. Either these equations are fulfilled or they lead to a certain number $K_{1}$ of new constraints

$$
\phi_{k} \approx 0 \quad, \quad k=M+1, \ldots, M+K_{1} \equiv J_{1},
$$


called secondary constraints. The primary and secondary constraints $\phi_{j} \approx$ $0, j=1, \ldots, J_{1}$ define a hypersurface $\Gamma_{1} \subseteq \Gamma_{p}$.

Now one has to check consistency for the primary and newly generated secondary constraints on $\Gamma_{1}$,

$$
\dot{\phi}_{j}=\left\{\phi_{j}, H\right\}+\left\{\phi_{j}, \phi_{n}\right\} u^{n} \equiv h_{j}+C_{j n} u^{n}=0 \quad \text { on } \quad \Gamma_{1}
$$

with the rectangular $J_{1} \times M$ matrix $C$. The left null-eigenvectors $w_{a}^{j}$ of $C_{j n}$ imply further conditions $w_{a}^{j} h_{j}=0$ on $\Gamma_{1}$ and may lead to further, socalled tertiary constraints which, together with the primary and secondary constraints, define a hypersurface $\Gamma_{2} \subseteq \Gamma_{1}$, etc.

This procedure terminates after a finite number of iterations and the following situation is reached: There is a hypersurface $\Gamma_{c} \subset \Gamma$ defined by

$$
\phi_{j} \approx 0 \quad, \quad j=1, \ldots, M+K \equiv J .
$$

For every left null-eigenvector $w_{a}^{j}$ of the rectangular matrix $C_{j m}=\left\{\phi_{j}, \phi_{m}\right\}$ the conditions $w_{a}^{j}\left\{\phi_{j}, H\right\} \approx 0$ are fulfilled. For the multiplier fields there are the equations

$$
\left\{\phi_{j}, H\right\}+\left\{\phi_{j}, \phi_{m}\right\} u^{m} \approx 0
$$

where $\approx$ now means equality on $\Gamma_{c}$. We note that the primary constraints are merely consequences of the definition of the momenta, whereas we used the equations of motion to arrive at the secondary constraints 2 .

We make the same regularity assumptions on the full set of constraints $\phi_{j}$ defining $\Gamma_{c}$ as we made on the primary constraints $\phi_{m}$ defining $\Gamma_{p}$. Also, we assume that the rank of $C$ is constant on $\Gamma_{c}$.

\subsection{First and second class constraints}

The distinction between primary and secondary constraints will be of minor importance in the final form of the Hamiltonian theory. A different classification of contraints, namely into first and second class [18], will play a central part. Let $v_{a}$ be a basis of the kernel of $C$,

$$
\left\{\phi_{j}, \phi_{m}\right\} v_{a}^{m} \approx 0, a=1, \ldots, \operatorname{dim} \operatorname{Ker} C=M-\operatorname{rank} C .
$$

The general solution for the multipliers $u$ in 3.29 has then the form

$$
u=\tilde{u}+\mu^{a} v_{a}
$$

\footnotetext{
${ }^{2}$ in the sequel I call all non-primary constraints secondary
} 
where $\tilde{u}$ is a particular solution. We have separated the part of $u$ that remains undetermined by the consistency conditions. This part contains $M-\operatorname{rank} C$ free functions $\mu^{a}$.

The combinations of primary contraints

$$
\phi_{a}=v_{a}^{m} \phi_{m}
$$

weakly commute with all other constraints,

$$
\left\{\phi_{a}, \phi_{j}\right\} \approx 0 \quad, \quad j=1, \ldots, J .
$$

Moreover, since the $v^{a}$ form a base of $\operatorname{Ker} C$, the $\phi_{a}$ are a complete set of primary contraints with this property. This leads to the concept of first class functions and in particular first class constraints (FCC). A function $F(q, p)$ is said to be first class if its Poisson brackets with all constraints vanish weakly $\left(\right.$ on $\left.\Gamma_{c}\right)$,

$$
\left\{F, \phi_{j}\right\} \approx 0 \quad, \quad j=1, \ldots, J .
$$

The set of first class functions is closed under Poisson brackets [18]. This is proved as follows: if $F, G$ are first class, then according to theorem 1

$$
\{F, \phi\}=\phi^{\prime} \quad, \quad\{G, \phi\}=\phi^{\prime \prime}
$$

for any constraint $\phi$, where $\phi^{\prime}, \phi^{\prime \prime}$ are some linear combinations of the constraints. Using the Jacobi identity we have

$$
\{\{F, G\}, \phi\}=\{F,\{G, \phi\}\}-\{G,\{F, \phi\}\}=\left\{F, \phi^{\prime \prime}\right\}-\left\{G, \phi^{\prime}\right\} \approx 0 .
$$

In particular the constraints $\phi_{a}$ are a complete set of first class primary constraints (modulo squares of second class constraints). Also, as a result of the Dirac-Bergman algorithm $H_{p}$ is first class.

A function that is not first class is called second class. I use a notation adapted to this new classification. All primary and secondary FCC are denoted by $\gamma_{a}$. The remaining constraints are called second class constraints (SCC) and I denote them by $\chi_{\alpha}$.

The first property we need is that the matrix of SCC

$$
\Delta_{\alpha \beta}=\left\{\chi_{\alpha}, \chi_{\beta}\right\}
$$

is non-singular. Indeed, if it was singular, then there would exist a null vector $r^{\alpha} \Delta_{\alpha \beta} \approx\left\{r^{\alpha} \chi_{\alpha}, \chi_{\beta}\right\} \approx 0$. Since $r^{\alpha} \chi_{\alpha}$ also commutes weakly with 
the FCC (by their first class property) it would weakly commute with all constraints and would be first class which contradicts our assumption. For counting degrees of freedom it is important to note that the number of SCC must be even. Otherwise the antisymmetric $\Delta$ would be singular.

Now consider the consistency conditions 3.29. They are identically fulfilled for the $\gamma_{a}$. For the SCC we have

$$
\left\{\chi_{\alpha}, H\right\}+\Delta_{\alpha \beta} u^{\beta} \approx 0
$$

where $u^{\beta}=0$ if $\chi^{\beta}$ is a secondary SCC. Solving for the multipliers we obtain

$$
\Delta^{\beta \alpha}\left\{\chi_{\alpha}, H\right\}=\left\{\begin{array}{lll}
u^{\beta}, & \chi_{\beta} & \text { primary } \\
0, & \chi_{\beta} & \text { secondary }
\end{array}\right.
$$

where $\Delta^{\alpha \beta} \Delta_{\beta \gamma}=\delta_{\gamma}^{\alpha}$. Thus all multipliers belonging to the primary SCC are determined by the consistency conditions and we remain with the undermined multipliers $\mu^{a}$ in 3.31. We have the important result that the number of undetermined multipliers is equal to the number of independent primary FCC.

Inserting that into the equations of motion 3.23 we end up with

$$
\dot{F} \approx\{F, H\}+\left\{F, \phi_{a}\right\} \mu^{a}-\left\{F, \chi_{\alpha}\right\} \Delta^{\alpha \beta}\left\{\chi_{\beta}, H\right\},
$$

where the $\phi_{a}$ are the primary FCC. One can easily check that all constraints are preserved in time. 


\section{Chapter 4}

\section{Abelian Chern-Simons Theory with Sources}

To see how the general formalism works in an explicit example I consider the abelian Chern-Simons model [27, 37, 30, 22]. This is a field theory for a gauge potential $A_{a}$ in 3 space-time dimensions with coordinates $x=$ $\left(x^{0}, x^{1}, x^{2}\right) \equiv(t, \vec{x})$ with first order Lagrangean density

$$
\begin{aligned}
\mathcal{L} & =\frac{\kappa}{4} A^{a} \epsilon_{a b c} F^{c b}+A^{a} J_{a}, \quad \text { where } \\
F_{a b} & =\partial_{a} A_{b}-\partial_{b} A_{a} \quad, \quad \partial^{a} J_{a}=0 .
\end{aligned}
$$

Indices are lowered with the metric $\eta_{a b}=\operatorname{diag}(1,-1,-1)$ and $\epsilon_{a b c}$ is the LeviCivita symbol, $\epsilon_{012}=1$. We enclose the system in a finite box $[0, L] \times[0, L]$. The quantum theory is sensitive to the value of the coupling constant $\kappa$. For rational $2 \pi \kappa$ and vanishing external current $J$ the Hilberspace becomes finite-dimensional [37].

For arbitrary periodic currents the action is invariant under $U(1)$-gauge transformations

$$
A^{a} \rightarrow A^{a}+\partial^{a} \lambda \quad, \quad S \rightarrow S+\oint n^{a}\left(\frac{\kappa}{4} \lambda \epsilon_{a b c} F^{c b}+\lambda J_{a}\right)
$$

provided $\lambda$ vanishes at the initial and final times and $\lambda, F_{01}, F_{02}$ are periodic in $x^{1}, x^{2}$ with period $L$. So we shall assume that $\lambda$ and $F_{a b}$ are both periodic.

Since $\mathcal{L}$ is linear in the first derivatives the Hessian vanishes identically and the model is singular. Thus we expect 3 independent primary constraints (per space point). More explicitly, the canonical momentum densi- 
ties are

$$
\pi_{a}(\vec{x})=\frac{\delta L}{\delta \dot{A}^{a}(\vec{x})}=\frac{\kappa}{2} A^{b}(\vec{x}) \epsilon_{b a 0}
$$

and immediately lead to the primary constraints

$$
\left\{\phi_{m}\right\}=\left\{\pi_{0}, \pi_{1}+\frac{\kappa}{2} A^{2}, \pi_{2}-\frac{\kappa}{2} A^{1}\right\} \quad, \quad m=1,2,3 .
$$

The canonical Hamiltonian becomes

$$
\begin{aligned}
H & =\int d \vec{x}\left(\pi_{a}(\vec{x}) \dot{A}^{a}(\vec{x})-\mathcal{L}\right)=\int d \vec{x} \mathcal{H} \\
& =-\int d \vec{x}\left(\frac{\kappa}{2} A^{a} \epsilon_{a b i} \partial^{i} A^{b}+A^{a} J_{a}\right) \quad, \quad i=1,2 .
\end{aligned}
$$

and the time evolution is determined by 3.23 with primary Hamiltonian

$$
H_{p}=\int d \vec{x} \mathcal{H}_{p} \quad, \quad \mathcal{H}_{p}=\mathcal{H}+u^{m} \phi_{m},
$$

and fundamental Poisson bracket

$$
\left\{A^{a}(\vec{x}), \pi_{b}(\vec{y})\right\}=\delta_{b}^{a} \delta(\vec{x}-\vec{y}) .
$$

Let us now see whether secondary constraints arise from the consistency conditions $\dot{\phi}_{m} \approx 0$. One computes

$$
\begin{aligned}
\dot{\phi}_{1}(\vec{x}) & =\int d \vec{y}\left\{\pi_{0}(\vec{x}), \mathcal{H}_{p}(\vec{y})\right\} \\
& =-\int d \vec{y}\left\{\pi_{0}(\vec{x}), \kappa A^{0}(\vec{y}) \epsilon_{0 j i} \partial^{i} A^{j}(\vec{y})+J_{0}(\vec{y}) A^{0}(\vec{y})\right\} \\
& =\int\left(\kappa \epsilon_{0 j i} \partial^{i} A^{j}(\vec{y})+J_{0}(\vec{y})\right) \delta(\vec{x}-\vec{y}) \\
& =\epsilon_{0 j i} \kappa \partial^{i} A^{j}(\vec{x})+J_{0}(\vec{x})
\end{aligned}
$$

leading to the secondary constraint

$$
\phi_{4}(\vec{x})=\kappa F_{12}-J_{0}(\vec{x}) .
$$

Of course there is a quicker way to arrive at this conclusion, since $\dot{\phi}_{1}=$ $-\partial \mathcal{H}_{p} / \partial A^{0}$.

The time derivative of the other two primary constraints are 


$$
\begin{aligned}
\dot{\phi}_{2}(\vec{x}) & =\kappa\left(u^{3}-\partial^{2} A^{0}(\vec{x})\right)+J_{1}(\vec{x}) \\
\dot{\phi}_{3}(\vec{x}) & =\kappa\left(-u^{2}+\partial^{1} A^{0}(\vec{x})\right)+J_{2}(\vec{x})
\end{aligned}
$$

and putting them weakly to zero fixes the multipliers $u^{2}, u^{3}$. Finally, we must have

$$
\dot{\phi}_{4}(\vec{x})=\kappa \partial^{2} u^{2}(\vec{x})-\kappa \partial^{1} u^{3}(\vec{x})+\partial_{0} J_{0}(\vec{x}) \approx 0 .
$$

Inserting $u^{2}, u^{3}$ from 4 this becomes $\partial_{a} j^{a}=0$ and yields no further condition. Thus the Dirac-Bergman algorithm leads to 3 primary and 1 secondary constraint.

Obviously $\phi_{1} \equiv \gamma_{1}$ is first class. Also the combination

$$
\gamma_{2}=\partial_{1} \phi_{2}+\partial_{2} \phi_{3}+\phi_{4}=\partial_{i} \pi_{i}+\frac{\kappa}{2} F_{12}-J_{0}
$$

is first class and is the analog of the Gauss constraint in electrodynamics. As SCC we may take

$$
\chi_{1}=\phi_{2} \quad \text { and } \quad \chi_{2}=\phi_{3} \Longrightarrow \Delta_{\alpha \beta}=\kappa\left(\begin{array}{cc}
0 & 1 \\
-1 & 0
\end{array}\right) \delta(\vec{x}-\vec{y})
$$

$\Delta$ has inverse $\Delta^{-1}=-\Delta / \kappa$ and 3.40 reads

$$
\dot{F} \approx\{F, H\}+\left\{F, \gamma_{1}\right\} u^{1}+\frac{1}{\kappa} \int d \vec{y}\left\{F, \chi_{i}(\vec{y})\right\} \epsilon_{i j}\left\{\chi_{j}(\vec{y}), H\right\}
$$

Since the FCC commute with all constraints they generate transformations on $\Gamma_{c}$, i.e. if $(A, \pi)$ is on $\Gamma_{c}$ then

$$
\begin{aligned}
\delta_{\mathcal{N}} A(\vec{x}) & =\int d \vec{y}\left\{A(\vec{x}), \gamma_{a}(\vec{y})\right\} \mathcal{N}^{a}(\vec{y}) \\
\delta_{\mathcal{N}} \pi(\vec{x}) & =\int d \vec{y}\left\{\pi(\vec{x}), \gamma_{a}(\vec{y})\right\} \mathcal{N}^{a}(\vec{y})
\end{aligned}
$$

are variations tangent to it. This follows from $\delta_{\mathcal{N}} \phi_{j}=\int\left\{\phi_{j}, \gamma_{a}\right\} \mathcal{N}^{a} \approx 0$. Also, since these transformations commute with $H_{p}$, one expects that they are related to infinitesimal gauge transformations. Indeed, defining

$$
G=\int d \vec{y}\left(\partial_{0} \lambda(\vec{y}) \gamma_{1}(\vec{y})+\lambda(\vec{y}) \gamma_{2}(\vec{y})\right)
$$

one finds

$$
\delta_{\lambda} A^{a}(\vec{x})=\partial^{a} \lambda(\vec{x})
$$


Only a particular combination of the 2 FCC generate the gauge transformations 4.2 .

Contrary to the gauge generator $G$ the primary Hamiltonian contains only the primary FCC. A symmetric treatment of all FCC is achieved in the extended formalism 18 discussed below. We will come back to the important connection between FCC and gauge transformations in chap.7. 


\section{Chapter 5}

\section{The reduced phase space.}

First we shall consider SC systems for which no multipliers remain in the time evolution 3.40. There is no ambiguity in the dynamics. The term in 3.40 containing the inverse of $\Delta$ forces the system to stay on $\Gamma_{c}$. This surface will be the reduced phase space for SC systems.

In the second subsection we consider FC systems. These are the most important systems since all gauge theories are of this type. Gauge related point should be identified and this leads us to the problem of gauge invariant functions and/or the gauge fixing problem. The FCC together with a complete set of gauge fixing conditions form then a SC system. Thus for FC systems the gauge fixing define a subset $\Gamma_{r} \in \Gamma_{c}$ and this set is the reduced phase space.

\subsection{Second class constraints and Dirac bracket}

Motivated by 3.40 one introduces the Dirac bracket [17] for two phase space function as

$$
\{F, G\}^{*} \equiv\{F, G\}-\left\{F, \chi_{\alpha}\right\} \Delta^{\alpha \beta}\left\{\chi_{\beta}, G\right\},
$$

in terms of which

$$
\dot{F} \approx\{F, H\}^{*}
$$

for SC systems. This bracket possess the same properties as the Poisson bracket, i.e. they are antisymmetric, bilinear and obey the Jacobi identity and product rule. In addition we have

$$
\left\{F, \chi_{\alpha}\right\}^{*}=0 \quad, \quad\{F, G\}^{*} \approx\{F, G\} \quad, \quad\left\{F,\{G, K\}^{*}\right\}^{*} \approx\{F,\{G, K\}\}
$$


for arbitrary $F$ and first class $G, K$. These properties follow easily from the definition 5.1 and the property that first class functions have vanishing Poisson bracket with all constraints, e.g.

$$
\left\{F, \chi_{\alpha}\right\}^{*}=\left\{F, \chi_{\alpha}\right\}-\left\{F, \chi_{\beta}\right\} \Delta^{\beta \gamma}\left\{\chi_{\gamma}, \chi_{\alpha}\right\}=0 .
$$

Let us draw an immediate consequence of 5.4. According to theorem 1 any function can be replaced by its restriction to $\Gamma_{c}$, up to a linear combination of the constraints. Thus when calculating the Dirac bracket 5.1 between two functions we may replace them by their restriction to $\Gamma_{c}$ since the other brackets vanish on account of 5.4. It follows that the SCC can be set equal to zero either before or after evaluating the Dirac bracket.

To understand the geometric meaning of SCC we recall some facts from symplectic geometry [4]:

In most cases the phase space $\Gamma$ is the cotangental bundle $T^{*} Q$ over the configuration space $Q$ and hence is equipped with a natural symplectic structure (a non-degenerate closed two-form)

$$
\omega=\omega_{\mu \nu} d x^{\mu} \wedge d x^{\nu}
$$

which, according to Darboux, can locally be written as

$$
\omega=d q^{i} \wedge d p_{i} .
$$

Given $\omega$ we can assign to a functions its corresponding Hamiltonian vector field as

$$
F \Longrightarrow X_{F} \quad \text { by } \quad i_{X_{F}} \omega=d F,
$$

where $i_{X}$ and $d$ are the interior and exterior derivatives, respectively. In local coordinates we find

$$
\omega_{\mu \nu} X_{F}^{\mu} Y^{\nu}=\omega\left(X_{F}, Y\right)=i_{X_{F}} \omega(Y)=d F(Y)=\partial_{\nu} F Y^{\nu}
$$

for any vector field $Y$, or

$$
X_{F}^{\mu}=-\omega^{\mu \nu} \partial_{\nu} F, \quad \text { where } \omega^{\mu \nu} \omega_{\nu \rho}=\delta_{\rho}^{\mu} .
$$

The Poisson bracket of two functions is

$$
\{F, G\}=-\partial_{\rho} F \omega^{\rho \sigma} \partial_{\sigma} G=\omega_{\mu \nu} \omega^{\mu \rho} \partial_{\rho} F \omega^{\nu \sigma} \partial_{\sigma} G=\omega\left(X_{F}, X_{G}\right) .
$$

In particular, the change of $F$ under the Hamiltonian flow generated by $G$ can be written as

$$
F^{\prime} \equiv\{F, G\}=\omega\left(X_{F}, X_{G}\right)=i_{X_{F}} \omega\left(X_{G}\right)=d F\left(X_{G}\right)=X_{G}^{\mu} \partial_{\mu} F .
$$


In other words, the flows generated by $G$ are just the motions along the Hamiltonian vector field $X_{G}$. For $G=H 5.11$ are the Hamiltonian equations of motion.

Finally there is an important relation between Poisson and Lie bracket,

$$
\left[X_{F}, X_{G}\right]=-X_{\{F, G\}}, \quad \text { where }[X, Y]^{\mu}=X^{\alpha} \partial_{\alpha} Y^{\mu}-Y^{\alpha} \partial_{\alpha} Y^{\mu}
$$

are the Lie bracket. 5.12 is a consequence of the Jacobi identity.

Let us now return to the SC systems. The inclusion map $j: \Gamma_{c} \longrightarrow \Gamma$ induces a two-form on $\Gamma_{c}$, namely the pull back of the symplectic form $\omega$ on $\Gamma, \omega_{c}=j^{*} \omega$. $\omega_{c}$ is closed since $\omega$ has this property, but it may be degenerate. In this case it is called pre-symplectic. However, for SCC it is indeed symplectic, as follows from

Theorem 3 The $\chi_{\alpha}$ are second class if and only if $\omega_{c}=j^{*} \omega$ is nondegenerate.

Actually, we shall see that the Dirac bracket belongs to $\omega_{c}$. Most properties of the Dirac bracket, and in particular the Jacobi identity follow then at once from the corresponding properties of $\omega$.

To prove this theorem we must show that $\omega$ is non-degenerate on the vectors tangent to $\Gamma_{c}$. A vector field $Y$ is tangent to $\Gamma_{c}$ if $Y^{\mu} \partial_{\mu} \chi_{\alpha}$ vanishes for all constraints $\chi_{\alpha}$. With 5.11 this is equivalent to

$$
\omega\left(X_{\alpha}, Y\right) \approx 0 \quad \text { for all } \quad X_{\alpha} \equiv X_{\chi_{\alpha}} .
$$

On the other hand, from 5.10 follows that

$$
\omega\left(X_{\alpha}, X_{\beta}\right)=\left\{\chi_{\alpha}, \chi_{\beta}\right\} \equiv \Delta_{\alpha \beta} \not \approx 0
$$

so that the Hamiltonian vector fields generated by the constraints are not tangent. Let us now determine the vectors $X$ which obey

$$
\omega(X, Y) \approx 0 \text { for all tangent } Y .
$$

Since $\omega$ is non-degenerate 5.15 can have $\operatorname{dim} \Gamma-\operatorname{dim} \Gamma_{c}$ independent solutions $X$. But because of our regularity conditions on the constraints the $\operatorname{dim} \Gamma$ $\operatorname{dim} \Gamma_{c}$ Hamiltonian vector fields $X_{\alpha}$, which are not tangent, are independent solutions. Thus any $X$ which obeys 5.15 is a combination of the $X_{\alpha}$. Hence there cannot be a tangent $X$ obeying 5.15 which proves that $j^{*} \omega$ is nondegenerate. 
Note that we used the SC nature of the constraints and in particular that the flows generated by the SCC lead off the constraint surface.

Now it is easy to prove that the Dirac bracket furnishes an explicit representation for the induced Poisson bracket. For that consider

$$
\{F, G\}^{*}=\omega\left(X_{F}, X_{G}\right)-\omega\left(X_{F}, X_{\alpha}\right) \Delta^{\alpha \beta} \omega\left(X_{\beta}, X_{G}\right) \equiv \omega^{*}\left(X_{F}, X_{G}\right) .
$$

It is easy to see, that $\omega^{*}\left(X_{F}+X_{\chi}, X_{G}\right)=\omega^{*}\left(X_{F}, X_{G}\right)$ for any Hamiltonian vector field belonging to the constraints. Thus $\omega^{*}$ depends only on the tangent components of $X_{F}, X_{G}$. But for tangent $X_{F}$ we have $\omega\left(X_{F}, X_{\alpha}\right) \approx 0$ (see 5.13) and $\omega^{*}$ can be replaced by $\omega$ without changing the value of 5.16. This proves that $\omega^{*}$ is just the pull-back of $\omega$.

\subsection{First class constraints and gauge transforma- tions}

For purely FC systems the time evolution is governed by

$$
\dot{F} \approx\{F, H\}+\left\{F, \phi_{a}\right\} \mu^{a},
$$

where the $\phi_{a}$ are the primary FCC. For the same initial conditions we get different evolutions, depending on the multipliers $\mu^{a}$. The presence of arbitrary functions $\mu^{a}$ in the primary Hamiltonian tells us that not all $x=(q, p)$ are observable, i.e. there are several $x$ representing a given physical state. Assume that the initial value $x(0)$ is given and represents a certain state. Then the equation of motion should fully determine the physical state at later times. So if $x^{\prime}(t) \neq x(t)$ stem from the same physical state $x(0)$ they should be identified.

Consider two infinitesimal time evolutions of $F=F(0)$ given by $H_{p}$ with different values of the multipliers,

$$
F_{i}(t)=t\{F, H\}+t\left\{F, \phi_{a}\right\} \mu_{i}^{a} \quad \mathrm{i}=1,2 \quad .
$$

The difference $\delta F=F_{2}(t)-F_{1}(t)$ between the values is then

$$
\delta_{\mu} F=\left\{F, \mu^{a} \phi_{a}\right\}, \quad, \quad \mu=t\left(\mu^{2}-\mu^{1}\right) .
$$

Such a transformation does not alter the the physical state at time $t$, and hence is called gauge transformation [18]. Now we calculate

$$
\left[\delta_{\mu}, \delta_{\nu}\right] F=\left\{\left\{\mu^{a} \phi_{a}, \nu^{b} \phi_{b}\right\}, F\right\}
$$


and conclude that the commutator of any two primary FCC also generate gauge transformations. Also, performing a gauge transformation at $t=0$ with multipliers $\nu$ and then time evolve with multipliers $\mu$ should lead to the same state as doing these transformations in the reverse order. We find

$$
\left[\delta_{t, \mu}, \delta_{\nu}\right] F=-t\left\{\left\{\nu^{a} \phi_{a}, H\right\}, F\right\}-t\left\{\left\{\nu^{a} \phi_{a}, \mu^{b} \phi_{b}\right\}, F\right\}
$$

and conclude that the commutators $\left\{\phi_{a}, H\right\}$ also generates gauge transformations.

We have seen that the first class functions are closed with respect to the Poisson bracket and thus the $\left\{\phi_{a}, \phi_{b}\right\}$ and $\left\{\phi_{a}, H\right\}$ are linear combinations of the FCC. However, in general there will appear secondary FCC in these combinations. Also, if we compared the higher order terms in the time evolutions 5.18 we would find that time derivatives of $\left\{\phi_{a}, H_{p}\right\}$ generate gauge transformations. This way secondary FCC show up as gauge transformations in all relevant systems. Therefore, Dirac conjectured that all FCC $\gamma_{a}$ generate gauge transformations. We shall assume this conjecture to hold in what follows although there are some exotic counterexamples [2, 14.

Note, however, that if the structure constants in

$$
\left\{\gamma_{a}, \gamma_{b}\right\}=t_{a b}^{c} \gamma_{c}
$$

depend on the canonical variables, then $\left[\delta_{\mu}, \delta_{\nu}\right] F$ is a gauge transformation only on the constraint surface. Also, above we made the hidden assumption that time (or the space-time coordinates in field theory) is not affected by the transformation. Else we would have to take $F+\delta_{\mu} F$ at the transformed time $t+\delta_{\mu} t$ before calculating the second variation $\delta_{\nu}$. We come back to this point when discussing generally covariant theories in chap.7.

We conclude that the most general physically permissible motion should allow for an arbitrary gauge transformation to be performed during the time evolution. But $H_{p}$ contains only the primary FCC. We thus have to add to $H_{p}$ the secondary FCC multiplied by arbitrary functions. This led Dirac to introduce the extended Hamiltonian

$$
H_{p} \longrightarrow H_{e}=H+\mathcal{N}^{a} \gamma_{a}
$$

which contains all FCC [18]. $H_{p}$ accounts for all the gauge freedom.

Clearly, $H_{p}$ and $H_{e}$ should imply the same time evolution for the classical observables. Observables are gauge invariant functions on $\Gamma_{c}$, that is phase space functions that weakly commute with the gauge generators,

$$
F \quad \text { observable } \Longleftrightarrow\left\{F, \gamma_{a}\right\} \approx 0 \text { for all } a \text {. }
$$


Since $H_{e}-H_{p}$ is a combination of the secondary FCC, we have

$$
\dot{F} \approx\left\{F, H_{p}\right\} \approx\left\{F, H_{e}\right\}
$$

for any observable $F$, as required. In the extended formalism one makes no distinction between primary and secondary FCC since they are treated symmetrically. The introduction of $H_{e}$ is a new feature of the Hamiltonian scheme. It does not follow from the Lagrangean formalism.

Let us now investigate the geometric meaning of FC systems. As a preparation we show:

The induced 2-form $j^{*} \omega$ has rank $\geq N-2 M$, where $M$ is the number of independent first or second class constraints.

Let us assume that the tangent vectors $X_{p}, p=1, \ldots, P$ form a basis for the null-eigenvectors of $j^{*} \omega$, i.e. $j^{*} \omega\left(X_{p}, Y\right)=\omega\left(X_{p}, Y\right)=0$ for all tangent $Y$. Let us now see how big $P$ can be. For that we consider

$$
a^{p} \omega\left(X_{p}, Z_{q}\right)=0, \quad \text { where the } Z_{q}, \quad q=1, \ldots, M
$$

together with the tangent vectors form a basis of $T \Gamma$ at the point under consideration. These are $M$ equations for $P$ unknown. So, if $P \geq M$ then there would always exist a solution $X=a^{p} X_{p}$ with $\omega\left(X, Z_{q}\right)=0$ for all $Z_{q}$. Being also a null-eigenvector of $j^{*} \omega$ we would conclude that $\omega(X, Z)=0$ for all vectors $Z \in T \Gamma$ or that $\omega$ is degenerate. This then proves the statement above. Now we have the following

Theorem 4 For a FC system the induced two-form $j^{*} \omega$ is maximally degenerate. The kernel is spanned by the Hamiltonian vector fields belonging to the FCC.

First, if $X^{\mu} \partial_{\mu} \gamma_{b} \approx 0$ for all constraints, then $X$ is tangent. But since $X_{a}^{\mu} \partial_{\mu} \gamma_{a} \approx\left\{\gamma_{a}, \gamma_{b}\right\} \approx 0$, all Hamiltonian vectorfields belonging to the constraints are tangent. Second, for an arbitrary tangent vector $X$ we have $\omega\left(X_{a}, X\right)=i_{X_{a}}(X)=\partial_{\mu} \chi_{a} X^{\mu} \approx 0$. Thus the $M X_{a}$ 's are null-eigenvectors of the induced two-form and the rank of $j^{*} \omega$ equals $2 N-2 M$, i.e. it is maximally degenerate.

Thus we have the following situation: The $M$ FCC generate flows which stay on the constraint surface and which we identified with gauge transformations. The Hamiltonian vector fields belonging to the constraints are the null-directions of the induced pre-symplectic 2-form. That these null-vector fields generate gauge orbits follows from 
Theorem 5 On $\Gamma_{c}$ the vectors $X_{a}$ generate $M$-dimensional manifolds.

The proof uses the Frobenius integrability condition, which says that $M$ linearly independent vector fields are integrable (through each point in $\Gamma_{c}$ there is a surface, the gauge orbit, to wich the $X_{a}$ are tangent) iff all Lie brackets $\left[X_{a}, X_{b}\right]$ are linear combinations of $\left(X_{1}, \ldots, X_{M}\right)$. Indeed,

$$
\left[X_{a}, X_{b}\right]=-X_{\left\{\gamma_{a}, \gamma_{b}\right\}}=-t_{a b}^{c} X_{c}+\gamma_{c} \omega^{\mu \nu} \partial_{\nu} t_{a b}^{c} \approx-t_{a b}^{c} X_{c}
$$

where we used 5.22. Note that for $(q, p)$-dependent structure constant (as in gravity) the null vector fields are integrable only on the constraint surface.

In a next step one wants to eliminate the gauge degrees of freedom that is indentify points on the same gauge orbit. This can in principle be achieved by introducing gauge invariant variables, e.g. the transverse potential or holonomies in electrodynamics, or alternatively by fixing the gauge. A gauge fixing must obey two conditions: first it must be attainable and second it should fix the gauge uniquely 1 . We can fix the gauge by imposing the independent conditions

$$
F_{a}(q, p)=0, \quad \mathrm{a}=1, \ldots, \mathrm{M} . \quad,
$$

The surface defined by these conditions should intersect every gauge orbit in exactly one point. A necessary condition is that at least one gauge fixing function $F_{b}$ should change in the direction of all null-vectors $X_{a}$. In other words, there is at least one $F_{b}$ so that

$$
\lambda^{a}\left(X_{a}, \nabla F_{b}\right)=\lambda^{a}\left\{\gamma_{a}, F_{b}\right\} \neq 0
$$

for all $\lambda$. This implies that

$$
\operatorname{det}\left\{\gamma_{a}, F_{b}\right\} \equiv \operatorname{det} F_{a b} \neq 0 .
$$

In particular, if we could choose gauge fixings canonically conjugated to the constraints, $\left\{\gamma_{a}, F_{b}\right\}=\delta_{a b}$, then the gauge orbits would intersect the gauge fixing surfaces orthogonal and in this case $\operatorname{det} F_{a b}=1$. The determinant of $F$ plays an important part in the quantization of gauge systems and is the wellknown Fadeev-Popov determinant 20].

Because of 5.30 the FCC together with the gauge fixings form a SC system and we can take over the result from the previous subsection. The

\footnotetext{
${ }^{1}$ There may be obstructions to fulfilling these requirements as has been demonstrated by Gribov and Singer 26, 40].
} 
reduced phase space $\Gamma_{r}$ consists of the points fulfilling the constraints and gauge fixings. Collecting the $\gamma_{a}$ and $F_{a}$ into one vector, $\Omega_{p}, p=1, \ldots, 2 M$, we find for the Hamiltonian equation of motion for any phase space function

$$
\dot{F}=\{F, H\}-\left\{F, \Omega_{p}\right\} G^{p q}\left\{\Omega_{q}, H\right\} .
$$

\subsection{Mixed second and first class constraints}

Before gauge fixing the evolution is governed by the first class partner of the extended Hamiltonian

$$
H_{e}^{*}=H+\gamma_{a} \mathcal{N}^{a}-\chi_{\alpha} \Delta^{\alpha \beta}\left\{\chi_{\beta}, H\right\}
$$

since we have

$$
\dot{F}=\left\{F, H_{e}^{*}\right\}=\{F, H\}+\left\{F, \gamma_{a}\right\} \mathcal{N}^{a}-\left\{F, \chi_{\alpha}\right\} \Delta^{\alpha \beta}\left\{\chi_{\beta}, H\right\} .
$$

For a discussion of the starred variables see [11]. After gauge fixing one can again introduce starred variables with respect to the SC system $\psi_{I}=$ $\left(\chi_{\alpha}, \gamma_{a}, F_{b}\right)$. Denoting the Poisson brackets matrix of all these constraints $\psi_{I}$ by $\tilde{\Delta}$, we have

$$
\dot{F}=\left\{F, H^{*}\right\}, \quad \text { where } \quad H^{*}=H+\psi_{I} \tilde{\Delta}^{I J}\left\{\psi_{J}, H\right\} .
$$

\subsection{Gauge fixing of Chern-Simons theory}

In section 4 we have seen that the Chern-Simons Lagrangean 1 leads to two SCC and two FCC. Now we supplement those by two gauge fixing conditions, namely

$$
F_{1}=A_{0} \quad \text { and } \quad F_{2}=\partial_{i} A^{i} .
$$

Altogether the conditions $\left(\chi_{1}, \chi_{2}, \gamma_{1}, F_{1}, \gamma_{2}, F_{2}\right)$ form a SC system and define $\Gamma_{r}$. The surface defined by $\chi_{i}, \gamma_{1}$ and $F_{1}$ can be parametrized by the spatial components of the gauge potential which can be decomposed as

$$
A_{i}=\epsilon_{i j} \partial_{j} \varphi+\partial_{i} \lambda+\frac{1}{L} q_{i}
$$

with constant $q_{i}$ ?. Imposing further $\gamma_{2}$ and $F_{2}$ we see that

$$
A_{i}=-\frac{1}{\kappa} \epsilon_{i j} \partial_{j} \frac{1}{\triangle} J^{0}+\frac{1}{L} q_{i}
$$

\footnotetext{
${ }^{2}$ The $U(1)$-bundle over the torus is non-trivial and $A$ must be periodic only up to nontrivial gauge transformations [38]. For simplicity we assume here that $A$ is periodic and hence $\int F_{12}=0$
} 
so that $\Gamma_{r}=\left\{q_{1}, q_{2}\right\}$ is finite-dimensional. Furthermore, $\gamma_{2}=0$ and the periodicity of the $A_{i}$ imply that the total charge $Q=\int d \vec{x} J^{0}$ must vanish.

The inverse Poisson bracket 'matrix' reads

$$
\left(\tilde{\Delta}^{I J}\right)(x, y)=\frac{1}{\kappa}\left(\begin{array}{cccccc}
0 & -1 & 0 & 0 & \frac{1}{\triangle} \partial_{2} & 0 \\
1 & 0 & 0 & 0 & -\frac{1}{\triangle} \partial_{1} & 0 \\
0 & 0 & 0 & \kappa & 0 & 0 \\
0 & 0 & -\kappa & 0 & 0 & 0 \\
\frac{1}{\triangle} \partial_{2} & -\frac{1}{\triangle} \partial_{1} & 0 & 0 & 0 & -\kappa \frac{1}{\triangle} \\
0 & 0 & 0 & 0 & \kappa \frac{1}{\triangle} & 0
\end{array}\right) \delta(x-y)
$$

and one finds the following Dirac bracket for the coordinates on $\Gamma_{r}$

$$
\left\{q_{i}, q_{j}\right\}=-\delta_{i j}
$$

Calculating the starred Hamiltonian, one needs to remember that for a periodic function one has $\triangle^{-1} \triangle f=f-V^{-1} \int f$. After some algebra one finds

$$
H^{*}=-\int d \vec{x}\left\{A^{0}\left(\kappa F_{12}-J_{0}\right)+\frac{1}{\kappa} J_{0} \frac{1}{\triangle} \epsilon_{i j} \partial_{i} J_{j}\right\}-\frac{1}{2} j_{i} q^{i}-\frac{1}{\kappa} \epsilon_{i j} j_{i} p_{j},
$$

where we have introduced the mean 'fluxed'

$$
j_{i} \equiv \frac{1}{L} \int d \vec{x} J_{i} \quad, \quad q^{i} \equiv \frac{1}{L} \int d \vec{x} A^{i} \quad \text { and } \quad p_{i} \equiv \frac{1}{L} \int d \vec{x} \pi_{i} .
$$

After imposing the constraints $\chi_{i}, \gamma_{2}$ and $F_{1}$ the non-trivial equations of motion take the simple form

$$
\dot{q}_{i}=-\frac{1}{\kappa} \epsilon_{i j} j_{j}
$$

Of course, the evolution belonging to $H^{*}$ stays on $\Gamma_{r}$. Since $\Gamma_{r}$ is 2dimensional the (topological) Chern-Simons theory is effectively a simple mechanical system. This was expected from the beginning since there are 6 constraints and gauge fixings for 6 degrees of freedom (per $\vec{x}$ ).

To see the meaning of this result more clearly, let us see what the observables are. As coordinates on $\Gamma_{c}$ we may take $\lambda$ and $q_{i}$ in 5.36, so that we are considering functionals $F\left[A^{0}, \lambda, q_{i}, J_{a}\right]$. Such $F$ commute with the FCC if they are independent of $\lambda$ and $A^{0}$. Hence observables have the form

$$
F=F\left[J_{a}, q_{i}\right]
$$


and depend only on the zero-modes of the $A_{i}$.

Let us finally remark that for a pure CS theory $(J=0)$ the Lagrangean density is invariant, up to a total time derivative, under global gauge transformations for which only $e^{i \lambda}$ must be periodic. This introduces global gauge transformations with windings around the handels of the torus (the box with opposite points identified). Hence we must identify gauge potentials which are related by such global gauge transformations

$$
A_{i} \sim A_{i}+\frac{2 \pi}{L} n_{i} \quad \text { or } \quad q_{i} \sim q_{i}+2 \pi n_{i} .
$$

Gauge invariant functionals must be invariant under such transformations. Thus they depend only on $\exp \left(i \sum m_{i} q_{i}\right)$. For pure Chern-Simons theories we have

$$
e^{i \sum m_{i} q_{i}}=W(C, A)=\exp \left\{i \oint_{C} A\right\}
$$

on the constraint surface $\left(F_{12}=0\right)$ if the loop $C$ winds $m_{i}$-times around the torus in the direction $i$. For a contractible loop $W(C, A)$ vanishes. Thus, observables have the form

$$
F(A)=F\left(e^{i \oint_{C} A}\right)
$$

Let $C, D$ be 2 loops which wind $m_{i}, n_{i}$-times around the torus in the direction $i$. We parametrize them by $x(\tau), y(s)$. We compute

$$
\oint_{C} \oint_{D}\{A(x(\tau)), A(y(s))\}=-\oint_{C} \oint_{D} \epsilon_{i j} \dot{x}^{i}(\tau) \dot{y}^{j}(s) d \tau d s=-\left(n_{1} m_{2}-n_{2} m_{1}\right) .
$$

Upon deformation of the curves the commutator is invariant and therefore is a topological invariant. This can be understood by noting that for $J=0$ the Chern-Simons model 1 is invariant under space-time diffeomorphisms. In particular the spatial ones are generated by

$$
G_{d i f f}=\int d \vec{x} \epsilon^{i} A_{i} \gamma_{2}
$$

and hence observables must be invariant under spatial diffeomorphisms.

Finally note, that for the pure Chern-Simons theory the phase space variables $q_{i}$ lie in $[0,2 \pi]$, that is $\Gamma_{r}$ is compact and as a consequence the Hilberspace becomes finite dimensional. 


\subsection{First order action principle and symmetries}

The solutions to the primary Hamiltonian equations of motion 3.2, 3.22 extremize the primary (or total) first order action,

$$
\delta S_{p}=\delta \int_{t_{1}}^{t_{2}}\left(\dot{q}^{i} p_{i}-H-\sum_{\text {primary }} u^{m} \phi_{m}\right) d t=0
$$

with respect to variations $\delta q, \delta p, \delta u$ subject only to the restriction $\delta q\left(t_{1}\right)=$ $\delta q\left(t_{2}\right)=0$. The variables $u^{m}$ which have been introduced to make the Legendre transformation invertible appear now as Lagrange multipliers enforcing the primary constraints. It is clear that the theory is invariant under $H \rightarrow c^{m} \phi_{m}$ since such a change can be absorbed into the Lagrange multipliers.

The variational principle 5.49 is equivalent to

$$
\delta \int\left(\dot{q}^{i} p_{i}-H\right) d t=0 \quad \text { subject to } \quad \phi_{m}=\delta \phi_{m}=0 .
$$

There is yet another variational principle which for gauge invariant observables leads to the same time evolution, namely the extended action principle. The equations of motion for the extended formalism follow from

$$
\delta S_{e}=\delta \int\left(\dot{q}^{i} p_{i}-H-\sum_{\text {all constr. }} u^{j} \phi_{j}\right) d t=0,
$$

where the sum extends over all constraints.

Take the case of purely SCC and let $y^{i} \rightarrow x^{\mu}\left(y^{p}\right)$ be the imbedding of $\Gamma_{r} \subset \Gamma$. The Lagrange multiplier method guarantees that the implementation of the constraints $\chi_{\alpha}$, either directly or via the Lagrange multipliers, are equivalent. Now let us solve the constraint directly in 5.51. Recall that a symplectic 2 -form can locally be written as $\omega=d \theta$. The pull-back of the one-form potential $\theta$ is

$$
j^{*}(\theta)=j^{*}\left(p_{i} d q^{i}\right) \equiv j^{*}\left(a_{\mu} d x^{\mu}\right)=a_{\mu}(x(y)) \frac{\partial x^{\mu}}{\partial y^{p}} d y^{p} .
$$

Inserting this into the extended action one finds

$$
S_{e}[y]=\int\{\theta-H(x(y)) d t\}=\int\left(a_{p} \dot{y}^{p}-h\right) d t .
$$


The corresponding variational principle yield then the equation of motion for SC systems:

$$
\delta S_{e}[y]=0 \Longleftrightarrow \dot{y}^{p}=\left\{y^{p}, h(y)\right\}^{*} .
$$

This can be checked directly by using

$$
j^{*} \omega=j^{*} d \theta=d j^{*} \theta=d a_{p}(y) d y^{p} .
$$

The fact that 5.51 yields 5.53 is of practical use when calculating Dirac bracket. One solves for the constraints inside the action and from the new kinetic term one reads off the induced potential form on $\Gamma_{c}$. From 5.55 one computes the induced symplectic form and thus the Dirac bracket.

For FC systems it is also legitimate to solve the FCC inside the action

$$
S_{e}=\int\left(q^{i} p_{i}-H-\mathcal{N}^{a} \gamma_{a}\right) d t
$$

However, since the induced 2 form is degenerate the equations of motion on $\Gamma_{c}$ are not canonical. To get Hamiltonian equations one needs to go to $\Gamma_{r}$ by imposing additional gauge conditions. Then one may write down the corresponding action for the SC system as discussed above.

Let us demonstrate how this works for the Chern-Simons theory. Inserting the fields on the reduced phase space 5.37 into the first order action results in

$$
S=\int d t \int\left(\frac{\kappa}{2} q_{i} \epsilon_{i j} \dot{q}_{j}-q_{i} j_{i}\right)+\frac{1}{\kappa} \int d \vec{x} J^{0} \frac{1}{\triangle} \epsilon_{i j} \partial_{i} J_{j}
$$

which of course reproduces the correct equations of motion 5.42 . 


\section{Chapter 6}

\section{Yang-Mills Theories}

In this chapter I consider YM theories [1] without coupling to matter and emphasize the role of the constraints [28,42]. Pure non-abelian YM theories are interesting in their own right and they are highly non-trivial.

The YM action for the gauge fields is

$$
S=-\frac{1}{4} \int \operatorname{tr}\left[F_{\mu \nu} F^{\mu \nu}\right] d^{3} x d t
$$

where the field strength is Lie-algebra valued,

$$
F_{\mu \nu}=\partial_{\mu} A_{\nu}-\partial_{\nu} A_{\mu}-i\left[A_{\mu}, A_{\nu}\right] \quad, \quad A_{\mu}=A_{\mu}^{a} T_{a} \quad, \quad\left[T_{a}, T_{c}\right]=i f_{a b}^{c} T_{c},
$$

and the action is invariant under local gauge transformations

$$
A_{\mu} \longrightarrow e^{-i \theta} A_{\mu} e^{i \theta}+i e^{-i \theta} \partial_{\mu} e^{i \theta}
$$

with $\theta=\theta^{a} T_{a}$. The functions $\theta^{a}=\theta^{a}(x, t)$ are arbitrary functions on spacetime. The infinitesimal form of these gauge transformations is

$$
\delta_{\theta} A_{\mu}^{a}=-\left(\partial_{\mu} \theta^{a}+f_{b c}^{a} A_{\mu}^{b} \theta^{c}\right)=-\left(D_{\mu} \theta\right)^{a} .
$$

The local gauge invariance implies generalized Bianchi identities

$$
D_{\mu} D_{\nu} F^{\mu \nu}=0
$$

and renders the system singular. Among the field equations $D_{\mu} F^{\mu \nu}$ there are some containing second time-derivatives of $A$,

$$
D_{\mu} F^{\mu i}=0 \quad, \quad i=1,2,3
$$

\footnotetext{
${ }^{1} a, b, \ldots$ denote internal indices, $\mu, \nu \ldots$ space-time indices. The $T_{a}$ are hermitean generators and the structure constants $f_{a b}^{c}$ are totally antisymmetric.
} 
and which therefore are dynamical equations of motion. The others

$$
D_{\mu} F^{\mu 0}=D_{i} F^{i 0} \quad \text { or } \quad \phi_{m}(A, \dot{A})=\partial_{i} F_{m}^{i 0}+f_{a b}^{m} A_{i}^{a} F_{b}^{i 0}=0,
$$

where $m=1, \ldots, N=\operatorname{dim}$ (Gauge Group), are Lagrangean constraints. No further constraints appear since the time derivatives of the $\phi_{m}$ vanish on account of the field equations and the constraints themselves.

The canonical momenta conjugate to the $A$ 's are

$$
\pi_{a}^{\mu}=-F_{a}^{0 \mu} \quad, \quad\left\{A_{\mu}^{a}(\vec{x}), \pi_{b}^{\nu}(\vec{y})\right\}=\delta_{b}^{a} \delta_{\mu}^{\nu} \delta(\vec{x}-\vec{y}) .
$$

Since the field strength tensor is antisymmetric we obtain $N$ primary constraints

$$
\phi_{m}(A, \pi)=\pi_{m}^{0} \approx 0 .
$$

After a partial integration the canonical Hamiltonian is found to be

$$
H=\int d \vec{x}\left(\frac{1}{2} \pi_{i}^{a} \pi_{i}^{a}+\frac{1}{4} F_{i j}^{a} F_{i j}^{a}-A_{0}^{a} D_{i} \pi_{a}^{i}\right)
$$

and determines the time evolution

$$
\dot{F}=\left\{F, H_{p}\right\} \quad, \quad H_{p}=H+\int d \vec{x} u^{m} \phi_{m} .
$$

We need to check the consistency of the primary constraints:

$$
\dot{\phi}_{m}=\left\{\phi_{m}, H_{p}\right\}=0 \Longrightarrow \tilde{\phi}_{m}=\left(D_{i} \pi^{i}\right)_{m} \approx 0 .
$$

These $N$ secondary constraints are the generalizations of the Gauss constraint in electrodynamic.

The only non-trivial Poisson brackets of the algebra of constraints are

$$
\left\{\tilde{\phi}_{m}(\vec{x}), \tilde{\phi}_{n}(\vec{y})\right\}=f_{m n}^{p} \tilde{\phi}_{p}(\vec{x}) \delta(\vec{x}-\vec{y}) .
$$

The algebra is closed and therefore the $2 N$ constraints $\gamma_{a}=\left(\phi_{m}, \tilde{\phi}_{n}\right)$ form a FC system. Their Poisson brackets with $H$ are computed to be

$$
\left\{\phi_{m}, H\right\}=\tilde{\phi}_{m} \approx 0 \quad, \quad\left\{\tilde{\phi}_{m}, H\right\}=-f_{m n}^{p} A_{0}^{n} \tilde{\phi}_{p} \approx 0
$$

Let us now investigate the relation between the Hamiltonian gauge symmetries generated by the FCC and the Lagrangean gauge transformations 6.4. A general combination of the FCC $\phi=\int\left(\epsilon_{1}^{m} \phi_{m}+\epsilon_{2}^{m} \tilde{\phi}_{m}\right)$ generates the canonical symmetries 


$$
\begin{aligned}
\delta A_{\mu}^{a} & =\left\{\phi, A_{\mu}^{a}\right\}=\delta_{\mu}^{0} \epsilon_{1}^{a}-\delta_{\mu}^{i} D_{i} \epsilon_{2}^{a} \\
\delta \pi_{a}^{\mu} & =\left\{\phi, \pi_{a}^{\mu}\right\}=\delta_{i}^{\mu} f_{a b}^{c} \epsilon_{2}^{b} \pi_{c}^{i}+\int \phi_{m}\left\{\epsilon_{1}^{m}, \pi_{a}^{m}\right\},
\end{aligned}
$$

where we have already anticipated that $\epsilon_{1}$ depends on $A_{0}$. From 6.4 we read off how the $\epsilon$ 's must be chosen to correspond to Lagrangean gauge transformations. We find that the particular combination

$$
G=D_{0} \theta^{m} \phi_{m}-\theta^{m} \tilde{\phi}_{m}
$$

generates those transformations. Both primary and secondary FCC enter the Lagrangean gauge transformations similarly as for the CS theory.

Alternatively we can introduce gauge invariant variables, e.g. the Wilson loops [48], (see the lecture of R. Loll) or fix the gauge. To fix the gauge freedom we need $2 N$ gauge fixing conditions on the phase space variables $(A, \pi)$. Contrary to the situation in electrodynamics the gauge fixing in YM theories is rather subtle due to the Gribov problem [26]. Let $F_{a}\left(A_{\mu}\right)$ be local gauge fixings (which we assume not to depend on the momenta). Then the following problem may arise:

There are several field $A_{\mu}^{(j)}$ which are related by finite gauge transformations and all of them obey the gauge fixing.

This happens for example for $Q E D_{2}$ on the euclidean torus, if one decomposes a gauge field as in 5.36. The local condition $\partial^{\mu} A_{\mu}$ eliminates the gauge function $\lambda$ but does not constrain the $q_{i}$. But $2 \pi$ and $q_{i}+2 \pi$ are gauge equivalent configuration and this freedom cannot be fixed by a local gauge conditions. This is an example to a more general situation which has been proven by Singer [40]: For compactified YM-theories no global continuous gauge choice of the (local) form $F_{a}(A)=0$ exists which completely specifies the gauge.

Rather then dwelling on the various gauge fixings, their merits and drawbacks, which is of course important in a functional quantization, let me make some remarks about the variational problem.

The primary FCC $\phi_{m}$ are sort of uninteresting, since they can be easily eliminated in the extended formalism. We eliminate them by setting $\pi^{0}=$ $A_{0}=0$ and find the first order action

$$
S=\int\left[\pi_{a}^{i} \dot{A}_{i}^{a}-\mathcal{N}^{a} \gamma_{a}-\frac{1}{2}\left(\pi_{a}^{i} \pi_{i}^{a}+B_{a}^{i} B_{i}^{a}\right)\right] d t \quad, \quad \gamma_{a}=\left(D_{i} \pi^{i}\right)^{a},
$$


with multiplier fields $\mathcal{N}_{a}$. This form of the action is the one which is usually met in the literature (for example, in gravity one does not keep the momenta conjugated to the lapse and shift functions in the first order action). After having eliminated one pair of canonical variables one may wonder how one can reconstruct the full set of Lagrangean gauge transformation 6.4. This is indeed possible for all relevant systems in physics, e.g. YM-theories, the relativistic particle, string and gravity [35]. We will come back to this point in the next chapter. 


\section{Chapter 7}

\section{Lagrangean Symmetries of First-Class Hamiltonian Systems}

\subsection{The relativistic particle}

We describe the relativistic particle moving in 4-dimensional Minkowski spacetime by 4 scalar fields $\phi^{\mu}(t), \mu=0,1,2,3$, in 1-dimensional 'spacetime'. The action for the relativstic particle takes the form

$$
S=-\frac{1}{2} \int \sqrt{-g}\left[g^{00} \dot{\phi}^{\mu} \dot{\phi}_{\mu}+m^{2}\right] d t
$$

where the overdot denotes differentiation with respect to time $t$ and $\phi^{\mu} \phi_{\mu}=$ $-\left(\phi^{0}\right)^{2}+\sum_{1}^{3}\left(\phi^{i}\right)^{2}$. The $m^{2}$-term maybe viewed as 'cosmological constant' in 1-dimensional 'spacetime'.

$S$ is invariant with respect to general coordinate transformations (reparametrization invariance). The infinitesimal form of these transformations reads

$$
t \rightarrow t-\xi, \quad g_{00} \rightarrow g_{00}+\mathcal{L}_{\xi} g_{00}, \quad \phi^{\mu} \rightarrow \phi^{\mu}+\mathcal{L}_{\xi} \phi^{\mu}
$$

where $\mathcal{L}_{\xi}$ is the Lie-derivative. Introducing the lapse function $\mathcal{N}$ according to

$$
g_{00}=-\mathcal{N}^{2}
$$

we get the following transformation law from 7.2

$$
\delta \phi^{\mu}=\dot{\phi}^{\mu} \xi \quad \text { and } \quad \delta \mathcal{N}=(\mathcal{N} \xi)^{.} .
$$


The action 7.1 leads to the primary constraint $\phi_{1}=\pi_{g^{00}}=0$ which in turn implies the secondary one

$$
\phi_{2} \equiv \gamma=\frac{1}{2}\left(\pi^{\mu} \pi_{\mu}+m^{2}\right)
$$

These are FCC. The partial gauge fixing $F_{1}=g^{00}+1=0$ and $\phi_{1}$ form a conjugate second class pair and can be eliminated. Applying the standard procedure one finds then the following first order action

$$
S=\int\left[\pi_{\mu} \dot{\phi}^{\mu}-\mathcal{N} \gamma\right] d t
$$

where the $\pi_{\mu}$ are the momenta conjugated to the $\phi^{\mu}$,

$$
\left\{\phi^{\mu}, \pi_{\nu}\right\}=\delta_{\nu}^{\mu}
$$

The lagrangean multiplier $\mathcal{N}$ accompanying the constraint $\gamma$ (which is the super-hamiltonian) reintroduces the lapse function.

The action 7.6 is invariant with respect to the infinitesimal off mass-shell gauge transformations

$$
\delta_{\lambda} \phi^{\mu}=\left\{\phi^{\mu}, \lambda \gamma\right\}=\pi^{\mu} \lambda, \quad \delta_{\lambda} \pi_{\mu}=\left\{\pi_{\mu}, \lambda \gamma\right\}=0, \quad \delta_{\mathcal{N}}=\dot{\lambda} .
$$

With the identification $\lambda=\mathcal{N} \xi$ these transformations coincide with the diffeomorphism transformations (7.4), but only on mass shell:

$$
\dot{\phi}^{\mu}=\mathcal{N} \pi^{\mu}, \quad \dot{\pi}^{\mu}=0 .
$$

This is a general problem with diffeormorphism invariant theories. Only on-shell can the transformations generated by the FCC alone be identified with the Lagrangean symmetries. On the technical side the difficulty of identifying gauge and diffeomorphism transformations can be traced back to the nonlinear dependence of the constraint on the momentum. This is the important difference between internal and spacetime symmetries. In the following section we shall see how the canonical transformations generated by the FCC must be modified to yield all Lagrangean symmetries.

\subsection{Hamiltonian vs. Lagrangean symmetries}

In this chapter I shall consider a general FC system, the first order action of which is given by 5.56. These actions describes both systems with a finite 
or infinite number of degrees of freedom if the following condensed notation [16] is assumed: For systems with a finite number of degrees of freedom $a$ and $i$ are discrete and for field theories they denote both internal indices and space-coordinates. To distinguish internal from composite indices we shall use tildes for the latter ones. For field theories $\tilde{i}=\{i, \vec{x}\}$ and $\tilde{a}=\{a, \vec{x}\}$, where $i$ and $a$ are some discrete (internal) indices. For a scalar field $q^{\tilde{i}}(t)=$ $\varphi^{x}(t)=\varphi(x, t)$ and for a vector field $q^{\tilde{i}}(t)=A^{i, x}(t)=A^{i}(x, t)$. We adopt the Einstein convention and assume summation over discrete repeated indices and integration over continuous ones, for example

$$
\xi^{x} p_{i, x} \dot{q}^{i, x}=\sum_{i} \int d x \xi(x) p_{i}(x) \dot{q}^{i}(x)
$$

but

$$
\xi^{x} p_{i}^{x} q^{i x}=\sum_{i} \xi(x) p_{i}(x) q^{i}(x), \quad \text { no integration. }
$$

Also, we shall not distinguish $q^{i, x}$ and $q_{x}^{i}$ and use the position of the continuous index just to indicate when we should integrate. Sometimes it will be convenient to resolve the composite index $\tilde{i}$ (or $\tilde{a}$ ) as $i, x$ (or $a, x$ ). If the system contains fermions then some of the variables $p, q, \mathcal{N}$ will be of Grassmannian type.

In particular the first order action reads

$$
S_{e}=\int\left(p_{\tilde{i}} \dot{q}^{\tilde{i}}-\mathcal{N}^{\tilde{a}} \gamma_{\tilde{a}}-H\right) d t
$$

For FC systems the contraints and Hamiltonian form a closed algebra (possibly extended to fermionic variables, in which case the algebra is graded [13]):

$$
\left\{\gamma_{\tilde{a}}, \gamma_{\tilde{b}}\right\}=t_{\tilde{a} \tilde{b}}^{\tilde{c}} \gamma_{\tilde{c}} \quad \text { and } \quad\left\{H, \gamma_{\tilde{a}}\right\}=t_{\tilde{a}}^{\tilde{b}} \gamma_{\tilde{b}},
$$

with structure coefficients $t_{\tilde{a} \tilde{b}}^{\tilde{c}}$ and $t_{\tilde{a}}^{\tilde{b}}$. These coefficients may depend on the canonical variables $p, q$.

The equation of motion resulting from the variation of the action 7.12 with respect to $q, p$ and the Lagrangean multipliers $\mathcal{N}$ are

$$
\delta S_{e}=\int\left(\delta p_{\tilde{i}} E M\left(q^{\tilde{i}}\right)-\delta q^{\tilde{i}} E M\left(p_{\tilde{i}}\right)-\delta \mathcal{N}^{\tilde{a}} \gamma_{\tilde{a}}\right) d t+\text { bound. terms }
$$

are

$$
\begin{aligned}
E M\left(q^{\tilde{i}}\right) & \equiv \dot{q}^{\tilde{i}}-\left\{q^{\tilde{i}}, \mathcal{N}^{\tilde{b}} \gamma_{\tilde{b}}+H\right\}=0 \\
E M\left(p_{\tilde{i}}\right) & \equiv \dot{p}_{\tilde{i}}-\left\{p_{\tilde{i}}, \mathcal{N}^{\tilde{b}} \gamma_{\tilde{b}}+H\right\}=0 \\
\gamma_{\tilde{a}} & =0 .
\end{aligned}
$$


We use the abbreviations $E M(q)$ and $E M(p)$ for the equations of motion. Of course, on mass shell we have $E M=0$, but off mass shell either $E M(q)$ or $E M(p)$ (or both) does not vanish.

The first order action is invariant with respect to the infinitesimal transformations 35

$$
\hat{I}_{\xi, \lambda} F(q, p, \mathcal{N})=F\left(\hat{I}_{\xi, \lambda} q, \hat{I}_{\xi, \lambda} p, \hat{I}_{\xi, \lambda} \mathcal{N}\right), \quad \hat{I}_{\xi, \lambda}=\hat{1}+\delta_{\xi, \lambda}+\cdots
$$

where

$$
\begin{aligned}
\delta_{\xi, \lambda} q^{i x} & =E M\left(q^{i x}\right) \xi^{x}+\left\{q^{i x}, \lambda^{\tilde{b}} \gamma_{\tilde{b}}\right\}, \\
\delta_{\xi, \lambda} p_{i}^{x} & =E M\left(p_{i}^{x}\right) \xi^{x}+\left\{p_{i}^{x}, \lambda^{\tilde{b}} \gamma_{\tilde{b}}\right\} \\
\delta_{\xi, \lambda} \mathcal{N}^{\tilde{a}} & =\dot{\lambda}^{\tilde{a}}-\lambda^{\tilde{b}} \mathcal{N}^{\tilde{c}} t_{\tilde{c} \tilde{b}}^{\tilde{a}}-\lambda^{\tilde{b}} t_{\tilde{b}}^{\tilde{a}}
\end{aligned}
$$

The parameters $\xi^{x}=\xi(x, t)$ and $\lambda^{\tilde{a}}=\lambda^{a}(\mathcal{N}, x, t)$ are the parameters of the infinitesimal transformations. The first order Lagrangean is invariant up to a total time-derivative and correspondingly the action up to boundary terms

$$
\delta_{\xi, \lambda} S_{e}=\left.\left(p_{\tilde{i}} \delta_{\xi, \lambda} q^{\tilde{i}}-\lambda^{\tilde{a}} \gamma_{\tilde{a}}\right)\right|_{t_{i}} ^{t_{f}} .
$$

If the parameters $\xi, \lambda^{\tilde{a}}$ vanish at the initial and final times then $\delta_{\xi, \lambda} S=0$. If we would like the action to be invariant even under transformations with non-zero $\xi, \lambda$ at the boundaries then we need to add to the action the total derivative of some function $Q(q, p)$ which satisfies the equation

$$
\frac{\delta Q}{\delta q^{\tilde{i}}} \delta_{\xi, \lambda} q^{\tilde{i}}+\frac{\delta Q}{\delta p_{\tilde{i}}} \delta_{\xi, \lambda} p_{\tilde{i}}=\dot{\lambda}^{\tilde{a}} \gamma_{\tilde{a}}-p_{\tilde{i}} \delta_{\xi, \lambda} q^{\tilde{i}}
$$

where $\delta / \delta q$ etc. means functional derivative with respect to $q$.

To construct the finite transformations we need to apply the infinitesimal transformations many times. To be successful in this 'exponentiation' it is clear that the following necessary condition should be fulfilled: The algebra of infinitesimal transformations should be closed. To check the algebra of transformations let us calculate the result for the commutator of two subsequent infinitesimal transformations 7.16.7.17 with parameters $\xi_{1}, \lambda_{1}$ and $\xi_{2}, \lambda_{2}$ respectively. If we are making two subsequent infinitesimal transformations, then $\lambda^{\tilde{b}}$ of the second transformation will depend on $q, p$ if the structure constants depend on the canonical variables (since $\lambda^{\tilde{b}}$ depends on $\mathcal{N}$ ) and we should keep $\lambda^{\tilde{b}}$ inside the Poisson brackets even for purely bosonic theories. 
For an arbitrary algebraic function $F(q, p)$ of the canonical variables (for example $F=q$ or $F=p$ ) a rather lengthy but straightforward calculation yields the commutator

$$
\begin{gathered}
{\left[\hat{I}_{\xi_{2} \lambda_{2}}, \hat{I}_{\xi_{1} \lambda_{1}}\right] F^{x}(q, p)=\left(\frac{\delta F^{x}}{\delta q_{i}^{z}} \operatorname{EM}\left(q_{i}^{z}\right)+(q \rightarrow p)\right)\left(\dot{\xi}_{1}^{z} \xi_{2}^{z}-\xi_{1}^{z} \dot{\xi}_{2}^{z}\right)} \\
+\left(\left(\xi_{2}^{x}-\xi_{2}^{y}\right) \lambda_{1}^{\tilde{c}}-\mathcal{N}^{\tilde{c}} \xi_{2}^{x} \xi_{1}^{y}-(1 \leftrightarrow 2)\right)\left(\left\{F^{x}, \frac{\delta \gamma_{\tilde{c}}}{\delta q_{j}^{y}}\right\} \operatorname{EM}\left(q_{j}^{y}\right)+(q \rightarrow p)\right) \\
-\left(\xi_{2}^{x} \xi_{1}^{y}-\xi_{1}^{x} \xi_{2}^{y}\right)\left(\left\{F^{x}, \frac{\delta H}{\delta q_{j}^{y}}\right\} E M\left(q_{j}^{y}\right)+(q \rightarrow p)\right)+\left\{F^{x}, \bar{\lambda}^{\tilde{c}} \gamma_{\tilde{c}}\right\}
\end{gathered}
$$

and correspondingly for the Lagrangean multipliers one has

$$
\begin{array}{r}
{\left[\hat{I}_{\xi_{2} \lambda_{2}}, \hat{I}_{\xi_{1} \lambda_{1}}\right] \mathcal{N}^{\tilde{a}}=\left(\hat{I}_{\bar{\lambda}}-1\right) \mathcal{N}^{\tilde{a}}+\lambda_{2}^{\tilde{d}} \lambda_{1}^{\tilde{c}}\left(\dot{t}_{\tilde{c} \tilde{d}}^{\tilde{a}}-\left\{t_{\tilde{c} \tilde{d}}^{\tilde{a}}, \mathcal{N}^{\tilde{e}} \gamma_{\tilde{e}}+H\right\}\right)} \\
-\left(\lambda_{2}^{\tilde{c}} \xi_{1}^{x}-\lambda_{1}^{\tilde{c}} \xi_{2}^{x}\right)\left(\frac{\delta}{\delta q_{i}^{x}}\left(\mathcal{N}^{\tilde{b}} t_{\tilde{b} \tilde{c}}^{\tilde{a}}+t_{\tilde{c}}^{\tilde{a}}\right) E M\left(q_{i}^{x}\right)+(q \rightarrow p)\right),
\end{array}
$$

where we have introduced

$$
\bar{\lambda}^{\tilde{a}}=\lambda_{1}^{\tilde{e}} \lambda_{2}^{\tilde{b}} t_{\tilde{b} \tilde{e}}^{\tilde{a}}+\frac{\delta \lambda_{2}^{\tilde{a}}}{\delta \mathcal{N}^{\tilde{b}}} \delta_{\lambda_{1}} \mathcal{N}^{\tilde{b}}-\frac{\delta \lambda_{1}^{\tilde{a}}}{\delta \mathcal{N}^{\tilde{b}}} \delta_{\lambda_{2}} \mathcal{N}^{\tilde{b}}
$$

We would like to stress that when we are performing the second transformation in 7.207 .2 which follows the first one, then we must use the transformed variables. In particular, instead of $\lambda_{2}(\mathcal{N}, x, t)$ we must take $\lambda_{2}\left(\hat{I}_{\lambda_{1}} \mathcal{N}, x, t\right)$. This explains the appearence of the last terms in 7.22

In the particular case where the structure coefficents $t_{\tilde{b} \tilde{c}}^{\tilde{a}}$ do not depend on the canonical variables $q, p$ the parameter $\bar{\lambda}$ also does not depend on them as can be seen from 7.22 . Also, $\dot{t}_{\tilde{b} \tilde{c}}^{\tilde{a}}=0$ in this case and thus the commutator of two transformations generated by the constraints only $(\xi=0)$ yields again a transformation generated by the constraint. Hence, if the structure coefficients do not depend on the canonical variables the transformations generated by the constraints form a closed algebra off mass-shell. On the other hand, if the structure coefficients do depend on the canonical variables that does not automatically imply that the algebra of transformations will not close. Actually, the $q, p$-dependence in the formula 7.22 for $\bar{\lambda}$ can, in principle, be cancelled against an appropriate choice of the $\mathcal{N}$-dependence of $\lambda$. Actually this takes place for gravity, where some of the structure coefficients depend on $q$ [35]. Also the last terms in 7.2 vanish in this case on the hypersurface

$$
\mathcal{M}: \operatorname{EM}(q)=\dot{q}^{\tilde{i}}-\left\{q^{\tilde{i}}, \mathcal{N}^{\tilde{b}} \gamma_{\tilde{b}}+H\right\}=0
$$


and the algebra of transformations generated only by the constraints is closed on this hypersurface where the Lagrangean system lives. Although it is still closed off mass shell, it is not for all trajectories in phase space.

The algebra of transformations $7.20,7.2$ can also be closed in all relevant cases even when $\xi \neq 0$ if the $\lambda^{\tilde{b}}$ and $\xi$ are related in a certain way. The corresponding transformations are actually the symmetry transformations corresponding to the symmetries of the Lagrangean system when some of the constraints are nonlinear in the momenta.

Now we shall study the question when the symmetry transformations of a Hamiltonian system are also symmetry transformations of the corresponding Lagrangean system. For that the transformations 7.17 should at least leave the hypersurface $\mathcal{M}$ (see 7.23) on which the Lagrangean system lives, invariant. That is, they should leave any trajectory which belongs to the hypersurface $\mathcal{M}$ on this hypersurface. The necessary conditions for this can be gotten by varying 7.23 as follows

$$
\begin{aligned}
\left(\delta_{\xi, \lambda} q^{\tilde{i}}\right)^{\cdot} & =\frac{\delta^{2}\left(H+\mathcal{N}^{\tilde{e}} \gamma_{\tilde{e}}\right)}{\delta p_{\tilde{i}} \delta q^{\tilde{j}}} \delta_{\xi, \lambda} q^{\tilde{j}} \\
& +\frac{\delta^{2}\left(H+\mathcal{N}^{\tilde{e}} \gamma_{\tilde{e}}\right)}{\delta p_{\tilde{i}} \delta p_{\tilde{j}}} \delta_{\xi, \lambda} p_{\tilde{j}}+\left\{q^{\tilde{i}}, \delta_{\xi, \lambda} \mathcal{N}^{\tilde{e}} \gamma_{\tilde{e}}\right\}
\end{aligned}
$$

Thus the transformations $\delta q, \delta p$ and $\delta \mathcal{N}$ should satisfy the equation 7.2 on the hypersurface $\mathcal{M}$. Only in this case can the transformations in phase space be symmetry transformations of the corresponding Lagrangean system. Substituting 7.17 into 7.2 this condition simplifies to

$$
\frac{\delta^{2}\left(H+\mathcal{N}^{\tilde{e}} \gamma_{\tilde{e}}\right)}{\delta p_{i}^{x} \delta p_{j}^{y}} E M\left(p_{j}^{y}\right) \xi^{y}=\frac{\delta^{2} \gamma_{\tilde{e}}}{\delta p_{i}^{x} \delta p_{j}^{y}} E M\left(p_{j}^{y}\right) \lambda^{\tilde{e}}
$$

and imposes a certain functional dependence between $\xi$ and $\lambda^{a}$. If this condition is fulfilled the phase space transformations 7.17 can be interpreted as Lagrangean symmetries. At the same time the number of free functions becomes equal to the number of constraints as it should be.

Gauge Invariance. If the constraints are linear and $H$ at least quadratic in the momenta then only for $\xi^{z}=0$ can equation 7.25 be satisfied $\emptyset$. So, in this case the transformations which are generated by the constraints alone

\footnotetext{
${ }^{1}$ If $H$ and all constraints are at most linear in momenta, as it is the case for the Chern-Simons theories, then the Hamiltonian system is strongly degenerate.
} 
will also be symmetry transformations for the corresponding Lagrangean system. We shall call them gauge transformations. For example, in YangMills theories all constraints are linear in the momenta and as we have seen the finite gauge transformations can be recovered as transformations generated only by the constraints $(\xi=0)$ in the Hamiltonian formalism 9 .

Reparametrization invariance. Usually the reparametrization invariance of a Lagrangean system, if it exists, is identified with the gauge invariance in the Hamiltonian formalism. As we shall see they are actually very different and this identification can only be made on mass shell.

If some of the constraints in are nonlinear in momenta then it is obvious that the transformations generated by the constraints only $(\xi=0)$ do not satisfy the condition 7.25 and hence can not be symmetry transformations of the corresponding Lagrangean system. However, in all known theories with nonlinear constraints there are canonical coordinates such that $H=0$ and the condition 7.25 can be satisfied if we impose some functional dependence between $\lambda$ and $\xi$ in 7.17 so that $\xi \neq 0$ for such theories. Thus the nonlinear constraints generate the symmetry transformations of the corresponding Lagrangean system only in very special combination with 'trivial' $\xi$-transformations. The reason for that is the following: a transformation generated by a nonlinear constraints takes off mass-shell trajectories away from the subspace $\mathcal{M}$ and the extra compensating transformation returns the trajectories back to $\mathcal{M}$. More explicitly taking $\lambda^{\tilde{e}}$ to be $\lambda^{e z}=\mathcal{N}^{e z} \xi^{z}$ in 7.25 we reduce this equation to

$$
\mathcal{N}^{e z}\left(\xi^{y}-\xi^{z}\right) \frac{\delta^{2} \gamma_{e z}}{\delta p^{i x} \delta p^{j y}} E M\left(p^{j y}\right)=0 .
$$

One sees at once that if

$$
\frac{\delta^{2} \gamma_{e z}}{\delta p^{i x} \delta p^{j y}} \sim \delta(z-y)
$$

then even for constraints nonlinear in the momenta the equation 7.26 is satisfied off mass shell $(E M(p) \neq 0)$. From that it follows immediately that the transformations 7.17 with $\lambda^{e z}=\mathcal{N}^{e z} \xi^{z}$ are symmetry transformations for the corresponding Lagrangean system if $H=0$. We shall call the corresponding invariance reparametrization invariance: $\hat{R}_{\xi}=\hat{I}_{\xi, \lambda^{e z}=\mathcal{N} e z} \xi^{z}$. The

\footnotetext{
${ }^{2}$ Another interesting class of theories where all constraints are linear in momenta are the constrained Wess-Zumino-Novikov-Witten theories 21.
} 
explicit form of the reparametrization transformations read

$$
\begin{aligned}
\delta_{\xi} q^{i x} & =\dot{q}^{i x} \xi^{x}+\left(\xi^{y}-\xi^{x}\right)\left\{q^{i x}, \mathcal{N}^{b y} \gamma_{b y}\right\} \\
\delta_{\xi} p_{i}^{x} & =\dot{p}_{i}^{x} \xi^{x}+\left(\xi^{y}-\xi^{x}\right)\left\{p_{i}^{x}, \mathcal{N}^{b y} \gamma_{b y}\right\} \\
\delta_{\xi} \mathcal{N}^{a x} & =\left(\mathcal{N}^{a x} \xi^{x}\right)^{\cdot}-\xi^{y} \mathcal{N}^{b y} \mathcal{N}^{c z} t_{c z, b y}^{a x} .
\end{aligned}
$$

These transformations are the correct ones for theories with non-linear constraints. For example, for the relativistic particle the transformations 7.28 (and not the gauge transformations 7.8 generated by the first class constraints alone) coincide with 7.4 on $\mathcal{M}$.

For the relativistic particle, string and gravity the transformations 7.28 form a closed algebra off mass shell. This closure is not ment to be obvious since it depends on the concrete structure of the constraints. We verified it for each concrete system separately [35].

For the supersymmetric particle there are two constraints which are quadratic in the momenta and 7.28 is replaced by a two-parametric family of symmetry transformations. They are the supersymmetric extensions of the reparametrization transformations and do not coincide (off mass shell) with the gauge transformations generated by the constraints.

2An interesting question to which we have no general answer is the following: what are the conditions to exponentiate the infinitesimal transformations to finite ones. For the relativistic particle and string and for gravity the finite transformations for the corresponding Lagrangean systems are just the familiar symmetries. These finite symmetries can then be formulated in the Hamiltonian formulation and this way one can find the finite transformation in the first order formalism. But in general it is not clear whether the closing of the algebra of infinitesimal transformations is sufficient to make them finite. We suppose that this c2annot be the case since for a free nonrelativistic particle, which very probably does not admit any known finite local symmetry the transformations 7.17 with $\lambda=0$ form a closed algebra. This difficult and very important question (i.e. for the functional integral) what are the conditions such that the transformations can be made finite needs further investigation.

Constraints and the equations of motion. There is a very interesting and non-trivial connection between the equations of motion $\operatorname{EM}(q)=$ $E M(p)=0$ and the constraints $\gamma_{\tilde{a}}=0$. Clearly, since $\dot{\gamma}_{\tilde{a}}=0$ the classical trajectories will stay on $\Gamma_{c}$. Inversely, in some theories (e.g. gravity) we can get the equations of motions (or some of them as in string theory) if we only 
demand that the constraints are fulfilled for all $t$ (i.e. everywhere) and that the symmetry transformations do not destroy this property. For example, in gravity and string theory this means that we demand that the constraints are valid everywhere and for any choice of spacelike hypersurfaces, because the symmetry transformations (diffeomorphism transformations) can be intepreted as a change of foliation of space-time. It is very easy to arrive at this conclusion using the developed formalism. Let us consider how the constraints change under the symmetry transformations 7.17:

$$
\begin{aligned}
\delta_{\xi, \lambda} \gamma_{\tilde{a}} & =\frac{\delta \gamma_{\tilde{a}}}{\delta q^{i x}} \delta_{\xi, \lambda} q^{i x}+\frac{\delta \gamma_{\tilde{a}}}{\delta p_{i}^{x}} \delta_{\xi, \lambda} p_{i}^{x} \\
& =\frac{\delta \gamma_{\tilde{a}}}{\delta q^{i x}} E M\left(q^{i x}\right) \xi^{x}+\frac{\delta \gamma_{\tilde{a}}}{\delta p_{i}^{x}} E M\left(p_{i}^{x}\right) \xi^{x}+\lambda^{\tilde{c}} t_{\tilde{a} \tilde{c}}^{\tilde{b}} \gamma_{\tilde{b}}
\end{aligned}
$$

For the known theories the constraints are local in $q$ and $p$ and involve only space derivatives of $q$ up to second and $p$ up to first order. It follows then that the structure of the functional derivative of the constraints have the form

$$
\begin{aligned}
& \frac{\delta \gamma_{a y}}{\delta q^{i x}}=A_{i a} \delta(x, y)+B_{i a}^{j} \frac{\partial}{\partial y^{j}} \delta(x, y)+D_{i a}^{j k} \frac{\partial^{2}}{\partial y^{j} \partial y^{k}} \delta(x, y) \\
& \frac{\delta \gamma_{a y}}{\delta p_{i}^{x}}=E_{a}^{i} \delta(x, y)+F_{a}^{i j} \frac{\partial}{\partial y^{j}} \delta(x, y),
\end{aligned}
$$

where $A, B, \ldots$ are functions of $q^{y}$ and $p^{y}$. Substituting 9 into 9 a straightforward calculation yields

$$
\begin{aligned}
\delta_{\xi, \lambda} \gamma_{a y} & =\left(\dot{\gamma}_{a y}+\mathcal{N}^{\tilde{b}} t_{\tilde{b}, a y}^{\tilde{c}} \gamma_{\tilde{c}}+t_{a y}^{\tilde{c}} \gamma_{\tilde{c}}\right) \xi_{y} \\
& +\lambda^{\tilde{c}} t_{a y, \tilde{c}}^{\tilde{b}} \gamma_{\tilde{b}}+\left(B_{i a}^{j} E M\left(q^{i y}\right)+F_{a}^{i j} E M\left(p_{i}^{y}\right)\right) \frac{\partial \xi^{y}}{\partial y^{j}} \\
& +D_{i a}^{j k}\left(2 \frac{\partial E M\left(q^{i y}\right)}{\partial y^{j}} \frac{\partial \xi^{y}}{\partial y^{k}}+E M\left(q^{i y}\right) \frac{\partial^{2} \xi^{y}}{\partial y^{j} \partial y^{k}}\right) .
\end{aligned}
$$

It follows that for systems with a finite number of degrees of freedom $\delta \gamma \sim \gamma$, so that if we impose the constraints for a phase-space path then the transformed path satisfies also the constraints. For that none of the equations of motion must be satisfied. Thus for a finite number of degrees of freedom the constraints $\gamma_{a}$ do not tell us anything about the dynamics of the system.

Also, the gauge transformations $(\xi=0)$ do respect the constraints since $\delta_{\lambda, \xi=0} \gamma \sim \gamma$ as it is clear from 9. Again we see that the constraints together 
with the corresponding symmetry transformations tell us nothing about the dynamics.

However, for field theories with non-linear constraints we must take $\xi \neq 0$ in 7.17. In this case if not all of the coefficients $B, D$ and $F$ vanish we can get (some of) the equations of motion by demanding that the symmetry transformations respect the constraints, i.e. that $\delta \gamma=0$ if $\gamma=0$ everywhere. Whether some of the coefficients $B, D, F$ are non-zero depends on the concrete structure of the constraints. Only if the constraints contain some space derivatives of $q$ and/or $p$ are some of the coefficients non-zero. For example, in string theory only $B \neq 0$ and correspondingly only some equations of motion, namely two of the $E M(q)=0$ equations can be gotten by demanding that all constraints should be satisfied everywhere for any foliation. In gravity the situation is even more interesting, since all of the coefficients are non-zero and thus all equations of motion will be automatically satisfied if we demand just that $\gamma_{\tilde{a}}=0$ for any foliation. So the whole dynamics in gravity can be reduced to the requirement that the constraints are invariant under diffeomorphism transformations. 


\section{Bibliography}

[1] Albers, E.S. and Lee, B.W. (1973): Phys. Rep., 9C, 1

[2] Allcock, T.R. (1975): Phil. Trans. R. Soc. (Lond.), 279, 33

[3] Anderson, J.L and Bergman, P.G. (1951): Phys. Rev. 83, 1018

[4] Arnold, V. (1978): "Mathematical Methods of Classical Mechanics", Graduate Texts in Math. 60, Springer, Berlin

[5] Batalin, I.A. and Vilkovisky, G.A. (1977): Phys. Lett. 69B 309

[6] Becchi, C., Rouet C. and Stora, R. (1976): Ann. Phys. 98, 287; (1974):

Phys. Lett., B52, 344; (1975): Commun. Math. Phys., 42, 127

[7] Benguria, R., Cordero, P. and Teitelboim, C. (1977): Nucl. Phys., B122, 61

[8] Berezin, F.A. (1966): "The Method of Second Quantization", New York, Academic Press

[9] Berezin, F.A. and Marinov, M.S. (1977): Ann. Phys., 104, 336

[10] Bergman, P.G., Goldberg, I., Janis, A. and Newman, E. (1956): Phys. Rev. 103807

[11] Bergman, P.G. and Komar, A. (1960): Phys. Rev. Lett., 4, 432

[12] Bergman, P.G. (1961): Rev. Mod. Phys., 33, 510

[13] Casalbuoni, R. (1976): Nuovo Cimento, 33A, 115; (1976): Nuovo Cimento, 33A, 389

[14] Cawley, R. (1979): Phys. Rev. Lett., 42, 413 
[15] Chernoff, P. and Marsden, J. (1974): Lecture Notes Math., 425

[16] DeWitt, B. (1965): "Dynamical Theory of Groups and Fields", Gordon and Breach, New York

[17] Dirac, P.A.M. (1950): Can. J. Math., 2, 129; (1951): Can. J. Math., 3, 1; (1958): Proc. R. Soc. (Lond.), A246 326

[18] Dirac, P.A.M. (1964): "Lectures on Quantum Mechanics", Belfer Graduate School of Science, Yeshiva University, New York

[19] Fadeev L.D. (1969): Theor. Math. Phys., 11

[20] Fadeev, L.D. and Popov, V.N. (1967): Phys. Lett., 25B, 29

[21] Feher, L., O'Raifeartaigh, L., Ruelle, P., Tsutsui, I. and Wipf, A. (1992): Phys. Rep., 222, 1

[22] Fröhlich, J. and Studer, U. (1993): Rev. Mod. Phys., 65, 733

[23] Gervais, J.L. and Sakita, B. (1971): Nucl. Phys., B64, 632

[24] Gitman, D.M. and Tyutin, I.V. (1990): "Quantization of Fields with Constraints", Berlin, Springer

[25] Govaers, J. (1991): "Hamiltonian Quantisation and Constrained Dynamics", Leuven University Press

[26] Gribov, V.N. (1978): Nucl. Phys., B139, 1

[27] Hagen, C.R. (1984): Ann. Phys., 157, 342

[28] Hanson, A.J., Regge, T. and Teitelboim, C. (1976): "Constrained Hamilonian Systems", Accademia Nazionale dei Lincei

[29] Henneaux, M. and Teitelboim, C. (1992): "Quantization of Gauge Systems", Princeton Univ. Press

[30] Iengo, R. and Lechner, K. (1992): Phys. Rep., 213, 179

[31] Jackiw, R. (1988): Seminair de Mathematiques Superieurs, Montreal, Quebec

[32] Kiefer, C. and Wipf A. (1993): "Functional Schrödinger Equation for Fermions in External Gauge Fields", preprint ETH-TH/93-17 
[33] Kimura, T. (1962): Prog. Theor. Phys., 27, 747

[34] Marsden, J. (1974): "Applications of Global Analysis in Math. Physics", Publish or Perish, Boston.

[35] Mukhanov, V. and Wipf, A. (1992): "Unified Treatment of local Symmetries", preprint ETH-TH/92-49

[36] Polyakov, A.M. (1988): Mod. Phys. Lett., A3, 325

[37] Polychronakos, A.P. (1990): Ann. Phys., 203, 231

[38] Sachs, I. and Wipf, A. (1992): Helv. Phys. Acta, 65, 652

[39] Shanmugadhasan, S. (1963): Proc. Camb. Phil Soc., 59, 743

[40] Singer, I.M. (1978): Comm. Math. Phys., 60, 7

[41] Sudarshan, E.C.G. and Mukunda, N. (1974): "Classical Dynamics, A Modern Perspective", Wiley, New York

[42] Sundermeyer, K. (1982): "Constrained Dynamics", Springer Lecture Notes 169

[43] Symanzik, K. (1983): Nucl. Phys., 190 [FS3], 1

[44] Trautman, A. (1964): "Lectures on General Relativity", Brandeis Summer Institute in Theor. Phys., Prentice-Hall, N.J

[45] Tyutin, I.V. (1975): Lebedev preprint FIAN, 39

[46] Utiyama, R. (1959): Progr. Theor. Phys. Suppl., 9, 19

[47] Witten, E. (1989): Commun. Math. Phys., 121, 351

[48] Wu, T.T. and Yang, C.N. (1975): Phys. Rev., D12, 3845 